\title{
Classification of curves in 2D and 3D via affine integral signatures
}

\author{
S. Feng*, I. A. Kogan ${ }^{\dagger}$ H. Krim ${ }^{\ddagger}$
}

October 2, 2018

\begin{abstract}
We propose a robust classification algorithm for curves in 2D and 3D, under special and full groups of affine transformations. To each plane or spatial curve we assign a plane signature curve. Curves, equivalent under an affine transformation, have the same signature. The signatures introduced in this paper are based on integral invariants, which behave much better on noisy images than classically known differential invariants. The comparison with other types of invariants is given in the introduction. Though the integral invariants for planar curves were known before, the affine integral invariants for spatial curves are proposed here for the first time. Using the inductive variation of the moving frame method we compute affine invariants in terms of Euclidean invariants. We present two types of signatures, the global signature and the local signature. Both signatures are independent of parameterization (curve sampling). The global signature depends on the choice of the initial point and does not allow us to compare fragments of curves, and is therefore sensitive to occlusions. The local signature, although is slightly more sensitive to noise, is independent of the choice of the initial point and is not sensitive to occlusions in an image. It helps establish local equivalence of curves. The robustness of these invariants and signatures in their application to the problem of classification of noisy spatial curves extracted from a 3D object is analyzed.
\end{abstract}

\section{INTRODUCTION}

Curves and surfaces are fundamental entities in computer vision and pattern recognition. For example, the features of 3D or 2D objects are often spatial or planer curves, and their classification often reduces to a classification of curves under Euclidean, affine or projective transformations. A direct comparison of curves, such as shape matching, generally requires registration, and the ensuing complexity and difficulty in its application in many important problems, have recently led to a renewed research interest in transformation invariants.

Although geometric invariants have been applied to problems in computer image recognition and processing for decades [21, 22, 6, 29, 24], designing robust algorithms that are tolerant to noise and image occlusion remains an open problem. We start by providing a brief overview of various types of invariants that have appeared in computer vision literature. Euclidian differential

\footnotetext{
${ }^{*}$ ECE Department, North Carolina State University, Raleigh, NC, 27695-7914, E-mail: maxfeng@yahoo.com.

${ }^{\dagger}$ Department of Mathematics, North Carolina State University, Raleigh, NC, 27695-8205, E-mail: iakogan@ncsu.edu. Supported in part by NSF CCF-0728801 grant.

${ }^{\ddagger}$ ECE Department, North Carolina State University, Raleigh, NC, 27695-7914, E-mail: ahk@ncsu.edu. Supported in part by AFOSR F49620-98-1-0190 grant.
} 
invariants, such as Euclidean curvature and torsion for space curves, are the most classical. The affine and projective counterparts of curvature and torsion are well known. The dependence of curvature and torsion on high order derivatives (up to order 3 for the Euclidean group, 6 for the affine group and 9 for the projective group), makes numerical approximation of these invariants highly sensitive to noise, and therefore impractical in computer vision applications. This has motivated a high interest in other types of invariants such as semi-differential, or joint invariants [28, 23, 2] and various types of integral invariants [25, 13, 18, 20, 19. Integral invariants of a curve in the latter references depend on quantities obtained by integration of various functions along it. Since integration reduces the effect of noise, these invariants hold a clear advantage in practical applications.

While explicit expressions for integral invariants are known for plane curves in $2 \mathrm{D}$, they have thus far remained elusive for spatial curves in 3D, primarily on account of their computational complexity. With an increasing availability of 3D data acquisition systems and subsequent emerging applications, interest in 3D analysis and hence robust integral invariants for curves in 3D is becoming essential.

In [8] a hybrid integro-differential affine invariant which only uses first order derivatives along with integrals were computed. Although a performance improvement over classical differential invariants is obtained, the presence of first order derivatives still affects the performance.

In [9], we obtain for the first time explicit formulae of integral Euclidean and affine invariants for spatial curves in 3D. Hann and Hickman [13] introduced and computed these for plane curves. The type of integral invariants, computed in this paper, may be compared with moment invariants [27, 30]. We emphasize, however, the following difference: a moment invariant corresponds a number to a shape, whereas an integral invariant corresponds a curve to a curve. The standard action of the affine group on $\mathbb{R}^{3}$ induces an action on curves. Following the approach of [13] we prolong this action to certain integral expressions, called potentials, and then compute invariants that depend on these integral variables. A direct extension of [13] to 3D, using a Fels-Olver moving frame construction [7] is conceptually straightforward, but the computational complexity makes the problem intractable. An inductive implementation of the moving frame construction, proposed in [17], dramatically simplifies the algebraic derivations, as it allows one to construct invariants for the entire group from invariants of its subgroups: in our case affine invariants in terms of Euclidean ones.

The integral invariants defined in [13] and [9] are sensitive to parameterization, or sampling of the curve in the discrete case. A uniform parameterization is required for two curves to be compared. In order to overcome this limitation, we propose in this paper local and global $2 \mathrm{D} / 3 \mathrm{D}$ signatures for the special affine and full affine group. Signatures based on integral invariants are defined in an analogous way as signatures based on differential and joint invariants (see [3] for example). The global signature of a curve depends on the choice of its initial point and does not allow a comparison of its fragments. It is therefore sensitive to occlusions. The local signature, although slightly more computationally involved, is independent of the choice of the initial point and is not sensitive to the occlusion effects in the image. It allows to establish a local equivalence of curves being compared.

In Section 2, after reviewing the basic facts about group actions and invariants, we define the notion of integral jet bundle and integral invariants. Explicit formulae for affine integral invariants in terms of Euclidean for curves in 2D and 3D are given in Section 3, along with their geometric 
interpretation. In Section 4 we define a global integral signature which classifies curves with a given initial point up to affine transformations. We also define a local signature that is independent of the initial point of a curve. In Section 5 a discrete approximation of the signature construction is tested on curves extracted from $3 \mathrm{D}$ objects. The curves are given as discrete sequences of points, with possibly the additive noise. The experiments show that signature construction gives a robust method for classification of curves under affine transformations. The method can be easily adopted to a smaller Euclidean group.

\section{Group Action and Invariants}

In this section we review the basic terminology for the group actions and invariants, as well as the concept of prolonging the action to jet spaces and the notion of differential invariants. We then introduce the notion of integral jet space and define the corresponding prolongation of the action which gives rise to integral invariants.

\subsection{Definitions}

Definition 2.1 An action of a group $G$ on a set $S$ is a map $\alpha: G \times S \rightarrow S$ that satisfies the following two properties:

1. $\alpha(e, s)=s, \forall s \in S$, where $e$ is the identity of the group.

2. $\alpha\left(g_{1}, \alpha\left(g_{2}, s\right)\right)=\alpha\left(g_{1} g_{2}, s\right)$, for all $s \in S$ and $g_{1}, g_{2} \in G$.

For $g \in G$ and $s \in S$ we write $\alpha(g, s)=g \cdot s=\bar{s}$.

Definition 2.2 The orbit of a point $s \in S$ is the set $O_{s}=\{g \cdot s \mid g \in G\}$.

Definition 2.3 A function $f: S \rightarrow \mathbb{R}$ is called invariant if

$$
f(g \cdot s)=f(s), \forall g \in G \text { and } \forall s \in S .
$$

Invariant functions are constant along each orbit and can be used to find equivalence classes of objects undergoing various types of transformations.

Let $\mathcal{G} L(n)$ denote a group of non-degenerate $n \times n$ matrices with real entries. Its subgroup of matrices with determinant 1 is denoted by $\mathcal{S} L(n)$. The orthogonal group is $\mathcal{O}(n)=\{A \in$ $\left.\mathcal{G} L(n) \mid A A^{T}=I\right\}$, while the special orthogonal group is $\mathcal{S O}(n)=\{A \in \mathcal{O}(n) \mid \operatorname{det} A=1\}$. The semi-direct product of $\mathcal{G} L(n)$ and $\mathbb{R}^{n}$ is called the affine group: $\mathcal{A}(n)=\mathcal{G} L(n) \ltimes \mathbb{R}^{n}$. Its subgroup $\mathcal{S} A(n)=\mathcal{S} L(n) \ltimes \mathbb{R}^{n}$ is called the special affine group. The Euclidean group is $\mathcal{E}(n)=\mathcal{O}(n) \ltimes \mathbb{R}^{n}$. Its subgroup $\mathcal{S} E(n)=\mathcal{S} O(n) \ltimes \mathbb{R}^{n}$ is called the special Euclidean group.

In the paper we consider the action of the affine group $\mathcal{A}(n)$ and its subgroups on curves $\gamma(t)=\left(x_{1}(t), \ldots, x_{n}(t)\right), t \in[0,1]$ in $\mathbb{R}^{n}$ by a composition of a linear transformation and a translation, for $n=2$ and $n=3$ :

$$
\left(\begin{array}{c}
\overline{x_{i}(t)} \\
\vdots \\
\overline{x_{n}(t)}
\end{array}\right)=A\left(\begin{array}{c}
x_{1}(t) \\
\vdots \\
x_{n}(t)
\end{array}\right)+\left(\begin{array}{c}
v_{1} \\
\vdots \\
v_{n}
\end{array}\right) .
$$


where matrix $A \in \mathcal{G} L(n)$ defines a linear transformations and vector $\left(v_{1}, \ldots v_{n}\right) \in \mathbb{R}^{n}$ defines a translation.

\subsection{Prolongation of a group action}

Our goal is to obtain invariants that classify curves up to affine transformations. The classical method of obtaining such invariants is to prolong the action to the set of derivatives $\left\{x_{1}^{(k)}, \ldots, x_{i}^{(k)} \mid k=1 . . l\right\}$ of a sufficiently high order $l$

$$
\overline{x_{i}^{(1)}}(t)=\frac{d \overline{x_{i}}(t)}{d t}, \quad \overline{x_{i}^{(k+1)}}(t)=\frac{\overline{d x_{i}^{(k)}}(t)}{d t} .
$$

Definition 2.4 Functions of $\left\{x_{1}, \ldots, x_{n}, x_{i}^{(k)} \mid i=1 . . n, k=1 . . l\right\}$ that are invariant under the prolonged action (3) are called differential invariants of order $l$.

For the Euclidean action on curves in 3D, the two lowest order invariants are called curvature and torsion, and are classically known in differential geometry. Analogous invariants for the affine and projective groups are also known.

As noted in the introduction, differential invariants are highly sensitive to noise. We extend the approach of [13] from planar curves to curves in a space of arbitrary dimension. Let $\gamma(t)$ parametrized by $t \in[0,1]$ be a curve. We define integral variables

$$
x_{i}^{\left[\alpha_{1}, \ldots, \alpha_{n}\right]}(t)=\int_{0}^{t} x_{1}(t)^{\alpha_{1}} \cdots x_{n}(t)^{\alpha_{n}} d x_{i}(t),
$$

where the integrals are taken along the curve $\gamma(t)$ and $\alpha_{1}, \ldots, \alpha_{n}$ are non-negative integers, such that $\alpha_{1}+\cdots+\alpha_{i-1}+\alpha_{i+1}+\cdots+\alpha_{n} \neq 0$. We call $l=\alpha_{1}+\cdots+\alpha_{n}$ the order of integral variables, and there are totally $n\left(\frac{(n+l) !}{n ! l !}-(l+1)\right)$ of variables of order less or equal to $l$. Integration-by-parts formula dictates certain relations among the integral variables, the coordinates $x_{1}(t), \ldots x_{n}(t)$ of an arbitrary point on a curve $\gamma(t), t \in[0,1]$, and the coordinates $x_{1}^{0}, \ldots x_{n}^{0}$ of the initial point $\gamma(0)$. For example

$x_{1}^{[0,1,0, \ldots, 0]}(t)=\int_{0}^{t} x_{2}(t) d x_{1}(t)=x_{2}(t) x_{1}(t)-x_{2}^{0} x_{1}^{0}-\int_{0}^{t} x_{1}(t) d x_{2}(t)=x_{2}(t) d x_{1}(t)-x_{2}^{0} x_{1}^{0}-x_{2}^{[1,0,0, \ldots, 0]}(t)$.

It is not difficult to show that there are

$$
N_{l}=(n-1) \frac{(n+l) !}{n ! l !}-\sum_{m=1}^{n-1} \frac{(n-m+l) !}{(n-m) ! l !}
$$

independent integral variables of variables of order less or equal to $l$. A canonical choice of such variables is given by:

$$
x_{i}^{\left[\alpha_{j_{1}}, \ldots, \alpha_{j_{k}}\right]}(t)=\int_{0}^{t} x_{j_{1}}(t)^{\alpha_{j_{1}}} \cdots x_{j_{k}}(t)^{\alpha_{j_{k}}} d x_{i}(t), \text { where } j_{1}<j_{2}, \cdots<j_{k}, \alpha_{j_{1}}>0 \text { and } i>j_{1} \text {. }
$$

For example variable $x_{2}^{[1,0,0, \ldots, 0]}$ is canonical, but $x_{1}^{[0,1,0, \ldots, 0]}$ is not canonical. 
Definition 2.5 Let $\mathcal{I}^{l}$ be an $N_{l}$-dimensional space of independent integral variables of order $l$ and less, then the integral jet space of order $l$ (denoted $\mathcal{S}^{l}$ ) is defined to be a direct product of $\mathcal{I}^{l}$ and two copies of $\mathbb{R}^{n}$, i.e $\mathcal{S}^{l}=\mathcal{I}^{l} \times \mathbb{R}^{n} \times \mathbb{R}^{n}$. The coordinates $x_{1}, \ldots x_{n}$ of the first copy of $\mathbb{R}^{n}$ represent an arbitrary point on a curve $\gamma(t), t \in[0,1]$, and coordinates $x_{1}^{0}, \ldots x_{n}^{0}$ of the second copy of $\mathbb{R}^{n}$ represent the initial point $\gamma(0)$.

The action (2) can be prolonged to the curves on jet space as follows:

$$
\begin{aligned}
\left(\begin{array}{c}
\overline{x_{i}}(t) \\
\vdots \\
\overline{x_{n}}(t)
\end{array}\right) & =A\left(\begin{array}{c}
x_{1}(t) \\
\vdots \\
x_{n}(t)
\end{array}\right)+\left(\begin{array}{c}
v_{1} \\
\vdots \\
v_{n}
\end{array}\right) \\
\left(\begin{array}{c}
\overline{x_{i}^{0}} \\
\vdots \\
\overline{x_{n}^{0}}
\end{array}\right) & =A\left(\begin{array}{c}
x_{1}^{0} \\
\vdots \\
x_{n}^{0}
\end{array}\right)+\left(\begin{array}{c}
v_{1} \\
\vdots \\
v_{n}
\end{array}\right), \\
\overline{x_{i}^{\left[\alpha_{1}, \ldots, \alpha_{n}\right]}}(t) & =\int_{0}^{t}{\overline{x_{1}}}^{\alpha_{1}}(t) \cdots{\overline{x_{n}}}^{\alpha_{n}}(t) d \overline{x_{i}}(t) .
\end{aligned}
$$

It is important that the integration-by-parts relations among the integral variables are respected by the prolonged action, and therefore the action on the integral jet space is $\mathcal{S}^{l}$ is well defined.

Definition 2.6 A function on $\mathcal{S}^{l}$ which is invariant under the prolonged action (6) is called integral invariant of order $l$.

By introducing new variables

$$
X_{i}(t)=x_{i}(t)-x_{i}^{0}, i=1, \ldots, n
$$

and making the corresponding substitution into the integrals, we reduce the problem of finding invariants under the action (6) to an equivalent but simpler problem of finding invariant functions of variables $\left\{X_{1}, \ldots, X_{n}, X_{i}^{\left[\alpha_{1}, \ldots, \alpha_{n}\right]} \mid i=1 \ldots n\right\}$ under the action of $G L(n)$ defined by

$$
\begin{aligned}
\left(\begin{array}{c}
\overline{X_{i}}(t) \\
\vdots \\
\overline{X_{n}}(t)
\end{array}\right) & =A\left(\begin{array}{c}
X_{1}(t) \\
\vdots \\
X_{n}(t)
\end{array}\right) \\
\overline{X_{i}^{\left[\alpha_{1}, \ldots, \alpha_{n}\right]}}(t) & =\int_{0}^{t}{\overline{X_{1}}}^{\alpha_{1}}(t) \cdots{\overline{X_{n}}}^{\alpha_{n}}(t) d \overline{X_{i}}(t) .
\end{aligned}
$$

Invariants with respect to (6) may be obtained from invariants with respect to (8) by making substitution (7) 1 Invariants with respect to a very general class of actions of continuous finitedimensional groups on manifolds can be computed using Fels-Olver generalization [7] of Cartan's moving frame method (see also its algebraic reformulation [14]). The method consists of choosing a cross-section to the orbits and finding the coordinates of the projection along the orbits

\footnotetext{
1 This reduction by the group of translations can be put in the context of inductive method described in Appendix. We feel, however, that making this step "upfront" makes the presentation more transparent.
} 
of a generic point on a manifold to the cross-setion (see Appendix for more details). It can be, in theory, applied to find the invariants under the action described by (8) for arbitrary $n$. Hann and Hickman [13] used Fels-Olver method to compute integral invariants for planar curves $(n=2)$ under affine transformations and a certain subgroup of projective transformations. The corresponding derivation of invariants for spatial curves $(n=3)$ remained, however, out of reach due to computational complexity (it is often the case in the computational invariant theory that practical computations become unfeasible as the dimension of the group increases, despite the availability of a theoretical method to compute them [26, 5].) In [9], we derived, for the first time, integral invariants under the Euclidean and affine transformations for spatial curves using an inductive variation of the moving frame method [17, which allowes one to construct invariants for the entire group in terms of invariants of its subgroups: in our case, affine invariants in terms of Euclidean. Explicit derivation of invariants for curves of higher in the space of higher dimension $(n>3)$ remains an open problem, which seems at present, to be of more theoretical, than of practical interest.

\section{Integral invariants in $2 \mathrm{D}$ and $3 \mathrm{D}$}

In this section we present explicit formulas for integral invariants for $n=2$ (plane curves) and $n=3$ (spatial curves) under the affine action (6). The affine invariants are written in terms of the Euclidean invariants. We discuss their properties and geometric interpretation. The inductive derivation of these invariants is outlined in the Appendix.

\subsection{Integral Affine Invariants for Curves in 2D}

The standard affine group action on curves in $\mathbb{R}^{2}$ :

$$
\left(\begin{array}{l}
\bar{x}(t) \\
\bar{y}(t)
\end{array}\right)=\left(\begin{array}{ll}
a_{11} & a_{12} \\
a_{21} & a_{22}
\end{array}\right)\left(\begin{array}{c}
x(t) \\
y(t)
\end{array}\right)+\left(\begin{array}{l}
v_{1} \\
v_{2}
\end{array}\right), \quad \operatorname{det}\left(\begin{array}{ll}
a_{11} & a_{12} \\
a_{21} & a_{22}
\end{array}\right) \neq 0
$$

prolongs to the action on integral variables up to the third order.

By translating the initial point $\gamma(0)$ to the origin and making the corresponding substitution $X(t)=x(t)-x(0), Y(t)=y(t)-y(0)$ in the integrals, we reduce the problem to computing invariants under the action (8) with $n=2$. Among 12 integral variables

$$
\begin{aligned}
& X^{[i, j]}(t)=\int_{0}^{t} X(t)^{i} Y(t)^{j} d X(t), \quad j \neq 0, i+j \leq 3 \\
& Y^{[i, j]}(t)=\int_{0}^{t} X(t)^{i} Y(t)^{j} d Y(t), \quad i \neq 0, i+j \leq 3
\end{aligned}
$$

we make a canonical choice of 6 independent: $Y^{[1,0]}, Y^{[2,0]}, Y^{[1,1]}, Y^{[3,0]}, Y^{[2,1]}, Y^{[1,2]}$, as suggested by formula (5). The rest can be expressed in terms of those using integration by parts formulas, 
as follows:

$$
\begin{aligned}
X^{[0,1]} & =X Y-Y^{[1,0]} \\
X^{[0,2]} & =X Y^{2}-2 Y^{[1,1]} \\
X^{[1,1]} & =\frac{1}{2} X^{2} Y-\frac{1}{2} Y^{[2,0]} \\
X^{[1,2]} & =\frac{1}{2} X^{2} Y^{2}-Y^{[2,1]} \\
X^{[0,3]} & =Y^{3} X-3 Y^{[1,2]} \\
X^{[2,1]} & =\frac{1}{3} X^{3} Y-\frac{1}{3} Y^{[3,0]}
\end{aligned}
$$

This reduces the problem to finding invariants under the following $G L(2)$-action on $\mathbb{R}^{8}$. Denote $\operatorname{det} A:=a_{11} a_{22}-a_{21} a_{21}$. The action is defined by the following equations:

$$
\begin{aligned}
& \bar{X}=a_{11} X+a_{12} Y, \quad \bar{Y}=a_{21} X+a_{22} Y, \\
& \overline{Y^{[1,0]}}=(\operatorname{det} A) Y^{[1,0]}+\frac{1}{2} a_{11} a_{21} X^{2}+a_{12} a_{21} X Y+\frac{1}{2} a_{12} a_{22} Y^{2} \text {, } \\
& \overline{Y^{[1,1]}}=(\operatorname{det} A)\left(a_{22} Y^{[1,1]}+\frac{1}{2} a_{21} Y^{[2,0]}\right)+\frac{1}{3} a_{21}^{2} a_{11} X^{3}+\frac{1}{2} a_{21}\left(a_{11} a_{22}+a_{12} a_{21}\right) X^{2} Y \\
& +a_{21} a_{12} a_{22} X Y^{2}+\frac{1}{3} a_{22}^{2} a_{12} Y^{3} \\
& \overline{Y^{[2,0]}}=(\operatorname{det} A)\left(a_{11} Y^{[2,0]}+2 a_{12} Y^{[1,1]}\right)+\frac{1}{3} a_{11}{ }^{2} a_{21} X^{3}+a_{11} a_{12} a_{21} X^{2} Y+a_{12}^{2} a_{21} X Y^{2} \\
& +\frac{1}{3} a_{11} a_{12} a_{21} Y^{3} \\
& \overline{Y^{[1,2]}}=(\operatorname{det} A)\left(a_{22}^{2} Y^{[1,2]}+\frac{1}{3} a_{21}^{2} Y^{[3,0]}+a_{21} a_{22} Y^{[2,1]}\right) \\
& +\frac{1}{4} a_{11} a_{21}{ }^{3} X^{4}+\frac{1}{3} a_{21}^{2}\left(2 a_{11} a_{22}+a_{12} a_{21}\right) X^{3} Y \\
& +\frac{1}{2} a_{21} a_{22}\left(2 a_{12} a_{21}+a_{11} a_{22}\right) X^{2} Y^{2}+a_{12} a_{21} a_{22}{ }^{2} X Y^{3}+\frac{1}{4} a_{12} a_{22}{ }^{3} Y^{4}, \\
& \overline{Y^{[2,1]}}=(\operatorname{det} A)\left(\left(a_{11} a_{22}+a_{12} a_{21}\right) Y^{[2,1]}+2 a_{12} a_{22} Y^{[1,2]}+\frac{2}{3} a_{11} a_{21} Y^{[3,0]}\right) \\
& +\frac{1}{4} a_{11}^{2} a_{21}^{2} X^{4}+\frac{1}{3} a_{11} a_{21}\left(a_{11} a_{22}+2 a_{12} a_{21}\right) X^{3} Y+\frac{1}{2} a_{12} a_{21}\left(2 a_{11} a_{22}+a_{12} a_{21}\right) X^{2} Y^{2} \\
& +a_{12}^{2} a_{21} a_{22} X Y^{3}+\frac{1}{4} a_{12}^{2} a_{22}^{2} Y^{4}, \\
& \overline{Y^{[3,0]}}=(\operatorname{det} A)\left(a_{11}^{2} Y^{[3,0]}+3 a_{12}^{2} Y^{[1,2]}+3 a_{11} a_{12} Y^{[2,1]}\right) \\
& +\frac{1}{4} a_{11}^{3} a_{21} X^{4}+a_{11}^{2} a_{12} a_{21} X^{3} Y+\frac{3}{2} a_{11} a_{12}^{2} a_{21} X^{2} Y^{2}+a_{12}^{3} a_{21} Y^{3} X+\frac{1}{4} a_{12}^{3} a_{22} Y^{4} .
\end{aligned}
$$

We restrict the above action to the subgroup $\mathcal{S} O(2)$ of rotation matrices by setting $a_{11}=$ $\cos \phi, a_{12}=-\sin \phi, a_{21}=\sin \phi, a_{22}=\cos \phi$. We use the moving frame method to find invariants as described in the Appendix. Computationally this reduces to the substitution $a_{11}=\frac{X}{r}, a_{12}=$ 
$\frac{Y}{r}, a_{21}=\frac{-Y}{r}, a_{22}=\frac{X}{r}$, where $r=\sqrt{X^{2}+Y^{2}}$ in 11 . The resulting non-constant expressions comprise a set of generating invariants for the $S O(2)$ action:

$$
\begin{aligned}
X_{\mathcal{S} E} & =\sqrt{X^{2}+Y^{2}}=r \\
Y_{\mathcal{S E}}^{[1,0]} & =Y^{[1,0]}-\frac{X Y}{2}, \\
Y_{\mathcal{S} E}^{[1,1]} & =\frac{1}{r}\left(Y^{[1,1]} X-\frac{1}{2} Y^{[2,0]} Y-\frac{1}{6} X^{2} Y^{2}\right) \\
Y_{\mathcal{S E}}^{[2,0]} & =\frac{1}{r}\left(Y^{[2,0]} X+2 Y^{[1,1]} Y-\frac{1}{3} X^{3} Y-\frac{2}{3} X Y^{3}\right), \\
Y_{\mathcal{S E}}^{[1,2]} & =\frac{1}{r^{2}}\left(Y^{[1,2]} X^{2}-Y^{[2,1]} X Y+\frac{1}{3} Y^{[3,0]} Y^{2}-\frac{1}{12} X^{3} Y^{3}\right), \\
Y_{\mathcal{S} E}^{[2,1]} & =\frac{1}{r^{2}}\left(Y^{[2,1]}\left(X^{2}-Y^{2}\right)+2 Y^{[1,2]} X Y+\frac{2}{3} Y^{[3,0]} X Y-\frac{1}{4} X^{2} Y^{4}-\frac{1}{12} X^{4} Y^{2}\right), \\
Y_{\mathcal{S} E}^{[3,0]} & =\frac{1}{r^{2}}\left(Y^{[3,0]} X^{2}+3 Y^{[1,2]} Y^{2}+3 Y^{[2,1]} X Y-\frac{1}{4} X^{5} Y-\frac{3}{4} X^{3} Y^{3}-\frac{3}{4} X Y^{5}\right) .
\end{aligned}
$$

The invariants with respect to the special Euclidean group are obtained by making a substitution of $Y=y-y^{0}$ and $X=x-x^{0}$ in the above expressions (12): ${ }^{2}$ We note that since the denominators in the above formulas are invariant, the numerators are also invariant.

We use the inductive approach, described in the Appendix, to build invariants under the $S L(2)$-action defined by Eq. (11) with the condition $\operatorname{det} A=1$. The inductive method yields $\mathcal{S} A(2)$-invariants in terms of $\mathcal{S} E(2)$-invariants 12 :

$$
\begin{aligned}
Y_{\mathcal{S} A}^{[1,0]} & =Y_{\mathcal{S E}}^{[1,0]}=Y^{[1,0]}-\frac{X Y}{2} \\
Y_{\mathcal{S} A}^{[1,1]} & =X_{\mathcal{S E}} Y_{\mathcal{S} E}^{[1,1]}=Y^{[1,1]} X-\frac{1}{2} Y^{[2,0]} Y-\frac{1}{6} X^{2} Y^{2} \\
Y_{\mathcal{S} A}^{[1,2]} & =Y_{\mathcal{S} E}^{[1,2]} X_{\mathcal{S} E}^{2}=Y^{[1,2]} X^{2}-Y^{[2,1]} X Y+\frac{1}{3} Y^{[3,0]} Y^{2}-\frac{1}{12} X^{3} Y^{3} \\
Y_{\mathcal{S} A}^{[2,1]} & =Y_{\mathcal{S E}}^{[2,1]}-\frac{Y_{\mathcal{S} E}^{[2,0]}}{Y_{\mathcal{S} E}^{[1,1]}} Y_{\mathcal{S} E}^{[1,2]} \\
Y_{\mathcal{S} A}^{[3,1]} & =\frac{1}{X_{\mathcal{S E}}^{2}}\left(Y_{\mathcal{S} E}^{[3,0]}+\frac{3}{2} \frac{Y_{\mathcal{S E}}^{[2,0]}}{Y_{\mathcal{S} E}^{[1,1]}} Y_{\mathcal{S} E}^{[2,1]}+\frac{3}{4}\left(\frac{Y_{\mathcal{S E}}^{[2,0]}}{Y_{\mathcal{S E}}^{[1,1]}}\right)^{2} Y_{\mathcal{S E}}^{[1,2]}\right)
\end{aligned}
$$

By replacing $(X, Y)$ with $\left(x-x^{0}, y-y^{0}\right)$ in Eq. 13) we return to the integral jet space coordinates. In particular, $Y_{\mathcal{S} A}^{[1,0]}=Y^{[1,0]}-\frac{1}{2} X Y=\int_{0}^{t}\left(x-x^{0}\right) d y-\frac{1}{2}\left(x-x^{0}\right)\left(y-y^{0}\right)$.

\footnotetext{
${ }^{2}$ The notation for invariants suggests a certain correspondence between the invariants and the coordinate functions of the integral jet space, which we make clear in the Appendix.
} 
The following three special affine invariants are used in the next section to solve the classification problem with respect to both special and full affine groups:

$$
\begin{aligned}
& I_{1}=Y_{\mathcal{S} A}^{[1,0]}=Y^{[1,0]}-\frac{1}{2} X Y, \\
& I_{2}=Y_{\mathcal{S} A}^{[1,1]}=Y^{[1,1]} X-\frac{1}{2} Y^{[2,0]} Y-\frac{1}{6} X^{2} Y^{2}, \\
& I_{3}=Y_{\mathcal{S} A}^{[1,2]}=Y^{[1,2]} X^{2}-Y^{[2,1]} X Y+\frac{1}{3} Y^{[3,0]} Y^{2}-\frac{1}{12} X^{3} Y^{3} .
\end{aligned}
$$

To obtain invariants with respect to the full affine group we need to consider the effect of reflections and arbitrary scaling on the above invariants. We note that the transformation $x \rightarrow \lambda x$ and $y \rightarrow-\lambda y$ induces the transformation $I_{1} \rightarrow-\lambda^{2} I_{1}, I_{2} \rightarrow \lambda^{4} I_{2}$ and $I_{3} \rightarrow-\lambda^{6} I_{3}$. The following rational expressions are thus invariant with respect to the full affine group:

$$
\begin{aligned}
I_{2}^{\mathcal{A}} & =\frac{I_{2}}{I_{1}^{2}}=\frac{Y^{[1,1]} X-\frac{1}{2} Y^{[2,0]} Y-\frac{1}{6} X^{2} Y^{2}}{\left(Y^{[1,0]}-\frac{1}{2} X Y\right)^{2}} \\
I_{3}^{\mathcal{A}} & =\frac{I_{3}}{I_{1}^{3}}=\frac{Y^{[1,2]} X^{2}-Y^{[2,1]} X Y+\frac{1}{3} Y^{[3,0]} Y^{2}-\frac{1}{12} X^{3} Y^{3}}{\left(Y^{[1,0]}-\frac{1}{2} X Y\right)^{3}}
\end{aligned}
$$

The first of the above invariants is equivalent to the one obtained in [13].

\subsection{Geometric Interpretation of Invariants for Plane Curves}

The first two integral invariants (15) readily lend themselves to a geometric interpretation. Invariants $I_{1}$ is the signed area $B$ between the curve segment and the secant (see Figure 1). Indeed, the term $Y^{[1,0]}$ in the invariant $I_{1}$ is the signed area between the curve $\gamma(t)$ (whose initial point is translated to the origin) and the $Y$-axis, while $\frac{X Y}{2}$ is the signed area of the triangle $A$. Their difference is the area $B$. Since the $\mathcal{S} A(2)$ - action preserves areas, $I_{1}$ is clearly an invariant.

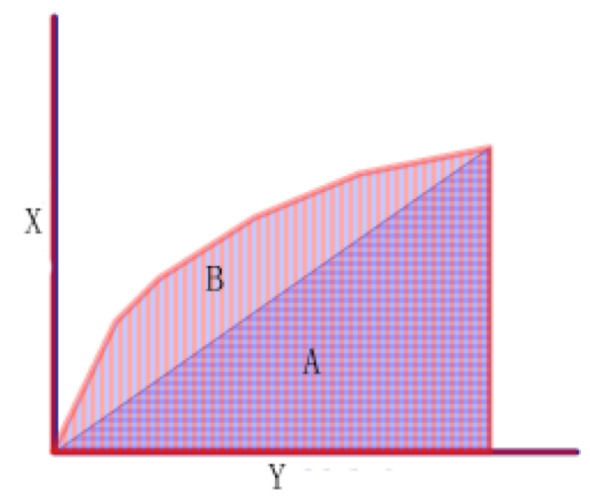

Figure 1: Geometric interpretation of the invariants $I_{1}$

The interpretation of $I_{2}$ is slightly more subtle. Using that $Y^{[2,0]}=X^{2} Y-2 X^{[1,1]}$ and rearranging the terms we rewrite $I_{2}$ as

$$
I_{2}=-\frac{1}{3}\left(\left(X^{2} Y^{2}-3 X Y^{[1,1]}\right)+\left(X^{2} Y^{2}-3 Y X^{[1,1]}\right)\right) \text {, where } X=x-x^{0}, Y=y-y^{0} .
$$




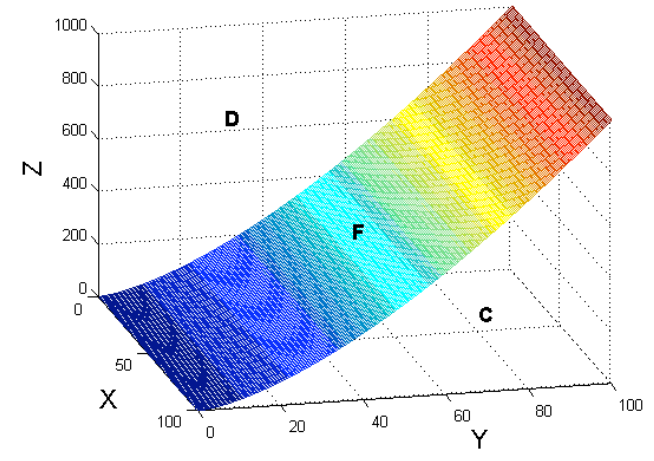

Figure 2: Geometric interpretation of the invariants $I_{2}$

Further, the curve $\gamma(t)$ is lifted from 2D to 3D by defining $z(t)=x(t) y(t)$ (similarly to the kernel idea), and (17) is rewritten as

$$
I_{2}=-\frac{1}{3}\left(\left(X Y Z-3 X \int_{0}^{t} Z d Y\right)+\left(X Y Z-3 Y \int_{0}^{t} Z d X\right)\right) \text { where } Z=X Y=\left(x-x^{0}\right)\left(y-y^{0}\right)
$$

The geometric meaning of $\left(X Y Z-3 X \int_{0}^{t} Z d Y\right)$ is illustrated in Figure 2, The term $\int_{0}^{t} Z d Y$ is the signed area "under" the plane curve $(Y(t), Z(t))$ in the $Y Z$-plane. Thus $X \int_{0}^{t} Z d Y$ is the signed volume $\mathrm{C}$ under the surface $F=\gamma(t) \times[0, X(t)]$ in Figure 2. Since $X Y Z$ is the signed volume of a rectangular prism $(\mathrm{C}+\mathrm{D}$ in Figure 2$)$, then $X Y Z-3 X \int_{0}^{t} Z d Y$ is the signed volume of the rectangular prism $(\mathrm{C}+\mathrm{D})$ minus three times the volume $\mathrm{C}$ "under" the surface $\gamma(t) \times[0, X(t)]$. Interchanging $X$ and $Y$ we obtain a similar interpretation for $X Y Z-3 Y \int_{0}^{t} Z d X$.

\subsection{Integral Affine Invariants for Curves in 3D}

The standard affine group action on curves in $\mathbb{R}^{3}$ :

$$
\left(\begin{array}{l}
\bar{x}(t) \\
\bar{y}(t) \\
\bar{z}(t)
\end{array}\right)=\left(\begin{array}{lll}
a_{11} & a_{12} & a_{13} \\
a_{21} & a_{22} & a_{23} \\
a_{31} & a_{32} & a_{33}
\end{array}\right)\left(\begin{array}{c}
x(t) \\
y(t) \\
z(t)
\end{array}\right)+\left(\begin{array}{c}
v_{1} \\
v_{2} \\
v_{3}
\end{array}\right), \quad \operatorname{det}\left(\begin{array}{lll}
a_{11} & a_{12} & a_{13} \\
a_{21} & a_{22} & a_{23} \\
a_{31} & a_{32} & a_{33}
\end{array}\right) \neq 0
$$

prolongs to the action to integral variables up to second order. We translate the initial point $\gamma(0)$ to the origin, and make the corresponding substitution $X(t)=x(t)-x(0), Y(t)=y(t)-$ $y(0), Z(t)=z(t)-z(0)$ in the integrals. This reduces the problem to computing $S L(3)$-invariants under the action (8) with $n=3$. Among 21 integral variables

$$
\begin{aligned}
& X^{[i, j, k]}(t)=\int_{0}^{t} X(t)^{i} Y(t)^{j} Z(t)^{k} d X(t), \quad j+k \neq 0, i+j+k \leq 2, \\
& Y^{[i, j, k]}(t)=\int_{0}^{t} X(t)^{i} Y(t)^{j} Z(t)^{k} d Y(t), \quad i+k \neq 0, i+j+k \leq 2, \\
& Z^{[i, j, k]}(t)=\int_{0}^{t} X(t)^{i} Y(t)^{j} Z(t)^{k} d Z(t), \quad i+j \neq 0, i+j+k \leq 2,
\end{aligned}
$$


we choose 11 independent: $X^{[1,1,0]}, X^{[1,0,1]}, X^{[0,2,0]}, Z^{[1,0,0]}, Y^{[1,0,0]}, Y^{[1,0,1]}, Z^{[0,1,0]}, Z^{[0,1,1]}, Z^{[0,2,0]}, Z^{[1,0,1]}, Z^{[1,1,0]}$. 3 The rest can be expressed in terms of those using the integration-by-parts formula. Using the inductive approach, we first compute the invariants with respect to rotations $S O(3)$. We find the following 8 independent invariants. We obtain $S E(3)$-invariants by replacing $(X, Y, Z)$ with $\left(x-x^{0}, y-y^{0}, z-z^{0}\right)$ ( See Appendix for details of the derivation.)

$$
\begin{aligned}
& X_{\mathcal{S E}}=\sqrt{X^{2}+Y^{2}+Z^{2}}, \\
& Z_{\mathcal{S} E}^{[0,1,0]}=\frac{X Y Z-2 X Z^{[0,1,0]}+2 Y Z^{[1,0,0]}-2 Z Y^{[1,0,0]}}{2 \sqrt{X^{2}+Y^{2}+Z^{2}}}, \\
& Y_{\mathcal{S E}}^{[1,0,0]}=\frac{Z_{R}^{[0,2,0]} Y_{R}^{[1,0,0]}+2 Z_{R}^{[0,1,1]} Z_{R}^{[1,0,0]}}{\sqrt{Z_{R}^{[0,2,0]^{2}}+4 Z_{R}^{[0,1,1]^{2}}}}, \\
& Y_{\mathcal{S} E}^{[1,0,1]}=-\frac{-2 Z_{R}^{[0,2,0]} Z_{R}^{[0,1,1]} Z_{R}^{[1,0,1]}-Z_{R}^{[0,1,1]} Z_{R}^{[0,2,0]} X_{R}^{[0,2,0]}+4 Z_{R}^{[0,1,1]^{2}} Z_{R}^{[1,1,0]}-Z_{R}^{[0,2,0]^{2}} Y_{R}^{[1,0,1]}}{Z_{R}^{[0,2,0]^{2}}+4 Z_{R}^{[0,1,1]^{2}}}, \\
& Z_{\mathcal{S} E}^{[0,2,0]}=\sqrt{Z_{R}^{[0,2,0]^{2}}+4 Z_{R}^{[0,1,1]^{2}}}, \\
& Z_{\mathcal{S} E}^{[1,0,1]}=-\frac{2 Z_{R}^{[0,2,0]} Z_{R}^{[0,1,1]} Z_{R}^{[1,1,0]}-Z_{R}^{[0,2,0]^{2}} Z_{R}^{[1,0,1]}+2 Z_{R}^{[0,1,1]^{2}} X_{R}^{[0,2,0]}+2 Z_{R}^{[0,1,1]} Z_{R}^{[0,2,0]} Y_{R}^{[1,0,1]}}{Z_{R}^{[0,2,0]^{2}}+4 Z_{R}^{[0,1,1]^{2}}}, \\
& Z_{\mathcal{S} E}^{[1,1,0]}=\frac{2 Z_{R}^{[0,2,0]} Z_{R}^{[0,1,1]} Z_{R}^{[1,0,1]}+Z_{R}^{[0,1,1]} Z_{R}^{[0,2,0]} X_{R}^{[0,2,0]}-4 Z_{R}^{[0,1,1]^{2}} Y_{R}^{[1,0,1]}+Z_{R}^{[0,2,0]^{2}} Z_{R}^{[1,1,0]}}{Z_{R}^{[0,2,0]^{2}}+4 Z_{R}^{[0,1,1]^{2}}},
\end{aligned}
$$

where expressions $Z_{R}^{[1,0,0]}, Z_{R}^{[0,1,0]}, Y_{R}^{[1,0,0]}, Z_{R}^{[0,1,1]}, Z_{R}^{[0,2,0]}, Z_{R}^{[1,0,1]}, Z_{R}^{[1,1,0]}, Y_{R}^{[1,0,1]}, X_{R}^{[1,1,0]}, X_{R}^{[1,0,1]}, X_{R}^{[0,2,0]}$ are provided at the end of the Appendix.

We use them to construct the following invariants with respect to the $S A(3)$ action.

$$
\begin{aligned}
X_{\mathcal{S} A} & =Z_{\mathcal{S} E}^{[0,1,0]} X_{\mathcal{S E}}, \\
Y_{\mathcal{S} A}^{[1,0,1]} & =\frac{2 Y_{\mathcal{S E}}^{[1,0,1]} Z_{\mathcal{S} E}^{[0,1,0]}-2 Z_{\mathcal{S E}}^{[0,1,0]} Z_{\mathcal{S E}}^{[1,1,0]}+3 Z_{\mathcal{S E}}^{[0,2,0]} Z_{\mathcal{S} E}^{[1,0,0]}}{2 Z_{\mathcal{S} E}^{[0,1,0]}}-\frac{1}{2} \\
Z_{\mathcal{S} A}^{[1,0,1]} & =\frac{Z_{\mathcal{S} E}^{[1,0,1]} Z_{\mathcal{S E}}^{[0,2,0]^{2}}}{Z_{\mathcal{S} E}^{[0,1,0]^{3}}} .
\end{aligned}
$$

We introduce a simpler notation for the special affine invariants which will subsequently be used

\footnotetext{
${ }^{3}$ The canonical choice dictated by $\sqrt{5}$ is $Y^{[1,0,0]}, Y^{[2,0,0]}, Y^{[1,1,0]}, Y^{[1,0,1]}, Z^{[1,0,0]}, Z^{[0,1,0]}, Z^{[0,1,1]}, Z^{[0,2,0]}, Z^{[2,0,0]}, Z^{[1,0,1]}, Z^{[1,1,0]}$. We made a computation with an equivalent but non-canonical set of variables.
} 
to solve the classification problem with respect to both the special and the full affine groups:

$$
\begin{aligned}
J_{1} & =X_{\mathcal{S} A}=n_{1} X+n_{2} Z-n_{3} Y \\
J_{2} & =-4\left(Y_{\mathcal{S} A}^{[1,0,1]}+\frac{1}{2}\right) X_{\mathcal{S A}}=2 n_{2}\left(X Y Z^{2}-3 Z^{[0,1,1]} X+3 Y Z^{[1,0,1]}-Z Z^{[1,1,0}-2 Z Y^{[1,0,1]}\right) \\
& +n_{3}\left(2 X Y^{2} Z+3 X Z^{[0,2,0]}-3 Z X^{[0,2,0]}-4 Y Z^{[1,1,0]}-2 Y Y^{[1,0,1]}\right) \\
& -2 n_{1}\left(3 Y X^{[1,0,1]}-3 Z X^{[1,1,0]}+X Z^{[1,1,0]}-X Y^{[1,0,1]}\right), \\
J_{3} & =\frac{27}{8} Z_{\mathcal{S} A}^{[1,0,1]} X_{S A}^{3},
\end{aligned}
$$

where $X=x-x^{0}, Y=y-y^{0}, Z=z-z^{0}$ and $n_{1}=\frac{Y Z}{2}-Z^{[0,1,0]}, n_{2}=\frac{X Y}{2}-Y^{[1,0,0]}, n_{3}=$ $\frac{X Z}{2}-Z^{[1,0,0]}$. The expression of the third invariant in terms of the original integral variables is too long to be included.

To obtain the invariants with respect to the full affine group we need to consider the effect of reflection and scaling on these invariants. For $\lambda \in \mathbb{R}$ scaling $(x, y, z) \rightarrow(\lambda x, \lambda y,-\lambda z)$ induces scaling $J_{1} \rightarrow-\lambda^{3} J_{1}, J_{2} \rightarrow \lambda^{6} J_{2}$ and $J_{3} \rightarrow \lambda^{6} J_{3}$. We therefore obtain the following two invariants with respect to the full affine group of transformations:

$$
J_{2}^{\mathcal{A}}=\frac{J_{2}}{J_{1}^{2}} \text { and } J_{3}^{\mathcal{A}}=\frac{J_{3}}{J_{1}^{2}} .
$$

\subsection{Geometric Interpretation of Invariants for Spatial Curves}

The first invariant $J_{1}$ may be viewed as an extension of the $2 \mathrm{D}$ invariant $I_{1}$ to $3 \mathrm{D}$. Indeed, $n_{1}$, $n_{2}$, and $n_{3}$ represent exactly the same area as the $2 \mathrm{D}$ invariant $I_{1}$ (in Figure 1 ) in three coordinate planes. They are extended from $2 \mathrm{D}$ area to $3 \mathrm{D}$ volume by multiplying by $X, Z$, and $Y$ respectively. For example, $n_{1} X$ is the volume $C$ under surface $F$ in Figure 2, and $n_{2} Z$ and $n_{3} Y$ are similar volumes obtained by relabelling of $X, Y, Z$ axis. Therefore, the invariant $J_{1}$ is the summation of two volumes $n_{1} X$ and $n_{2} Z$ minus the volume $n_{3} Y$. The geometric interpretation of the invariants $J_{2}$ and $J_{3}$, however, remains at the present time unclear to us.

\section{Curve Classification via Integral Signatures}

The integral invariants derived in the previous section depend on the choice of the initial point and the parameterization of a curve. For instance, consider a planar curve $\gamma(t)=(1 / 2 \sin t-$ $\cos t+1, \sin ^{2} t+\cos t-1$ ), shown in Figure 3-a. A curve $\bar{\gamma}(t)$ (Figure 3-b) is obtained from $\gamma(t)$ by a special affine transformation $\left(\begin{array}{cc}2 & 1 \\ 2 & 1.5\end{array}\right)$. A curve $\overline{\gamma_{a}(t)}$ (Figure $3-\mathrm{c}$ ) is obtained from $\gamma(t)$ by a full affine transformation $\left(\begin{array}{ll}2 & 2 \\ 4 & 3\end{array}\right)$.

The integral invariants $I_{1}$ and $I_{2}$ for curves in Fig. 3 a and Fig. 3 b with a matching parameterization coincide and are shown in Figure $4 \mathrm{a}$ and Figure $4 \mathrm{~b}$.

As illustrated in Figure $4 \mathrm{c}$ and Figure $4 \mathrm{f}$, these invariants change under reparametrization $\tau=\sqrt{t+1}$. Therefore the graph of invariants with respect to an arbitrary parameter can not be 
Figure 3:

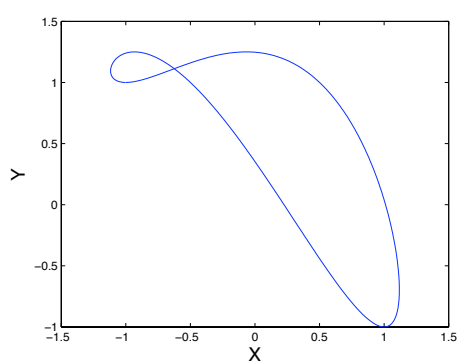

(a) curve $\gamma(t)$

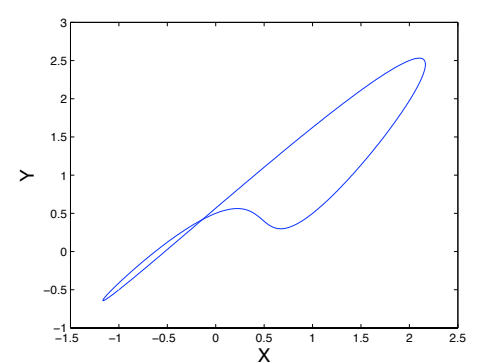

(b) special affine transformation $\bar{\gamma}(t)$

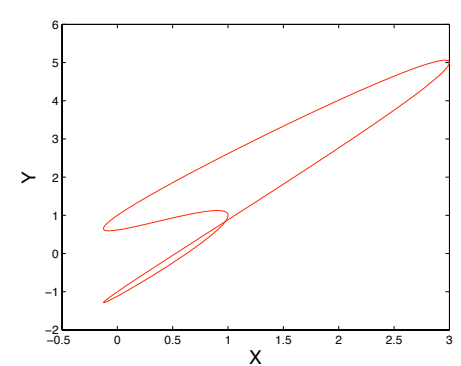

full affine transformation $\overline{\gamma_{a}(t)}$

used for curves comparison. In theory one can achieve a uniform affine invariant curve parameterization by using an affine analog of the Euclidean arc-length parameter $d \alpha=\kappa^{1 / 3} d s$, where $\kappa$ is Euclidean curvature and $d s$ is Euclidean arc-length. We would like however to keep our methods derivative free. Even when the uniform parameterization is achieved, the dependence of the invariants on the choice of the initial point presents another comparison challenge for matching closed curves, or for matching parts of the contours.

The signature construction, proposed in this section, leads to classification methods which are independent of parameterization and of the initial point. Inspired by signatures based on differential invariants [3, we use integral invariants to construct two types of signatures that classify curves under affine transformation: the global signature and the local signature. Global integral signature is independent of parametrization, but is dependent on the choice of the initial point and can not be used to compare partial contours. Local integral signature is independent of both the initial point and parametrization. They can be used to compare parts of the contours and therefore can be used on images with occlusions. As our experiments illustrate they are slightly more sensitive to noise than global signatures, but still provide robust classification results.

\subsection{Global Integral Affine Signature}

A global integral signature of a curve is the variation of one independent integral invariant, evaluated on the curve, relative to another. If a curve is mapped to another curve by a group transformation, their signatures coincide independently of the selected parametrization. The global signature, however, does depend on a choice of the initial point.

\subsubsection{Global affine signature for curves in 2D}

The special affine signature of a plane curve $\gamma(t)$ is constructed by, first, evaluating invariants $I_{1}$ and $I_{2}$ in Eq. 13 on this curve, and then plotting the parameterized curve $\left(I_{1}(t), I_{2}(t)\right)$ in $\mathbb{R}^{2}$. For instance, the signature of the planar curve $\gamma(t)$ shown on Figure 3 a is a plane curve in Figure 5 . Moreover, the signature of the curve $\overline{\gamma(t)}$, related to $\gamma(t)$ by an affine transformation (Figure 3 -b), as well as their reparametrization $\gamma(\tau)$ and $\overline{\gamma(\tau)}$ coincide with the signature of $\gamma(t)$.

Similarly, a full affine signature can be defined as a parameterized plane curve $\left(I_{2}^{\mathcal{A}}, I_{3}^{\mathcal{A}}\right)$ defined by invariants in Eq. 16). Alternatively we use the two special affine invariants to cancel the effects 


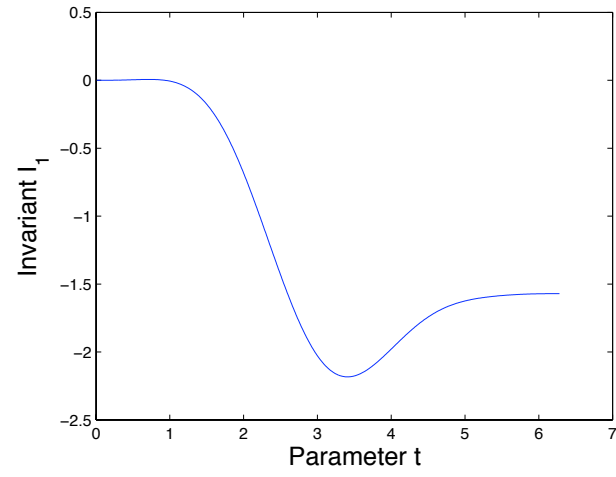

(a) Invariant $I_{1}$ for $\gamma(t)$ and $\overline{\gamma(t)}$

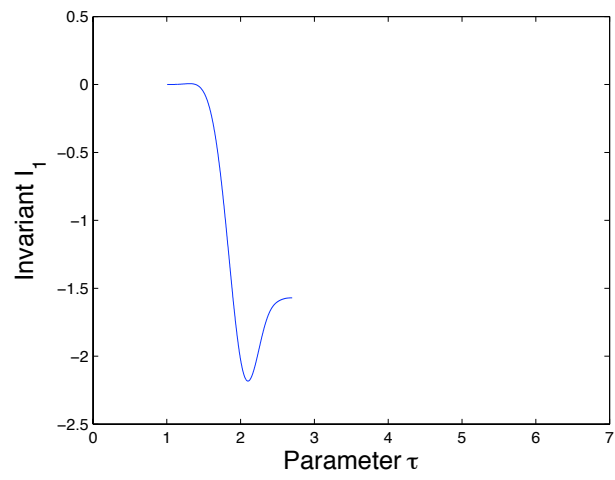

(c) Invariant $I_{1}$ for $\gamma(\tau)$ and $\overline{\gamma(\tau)}$

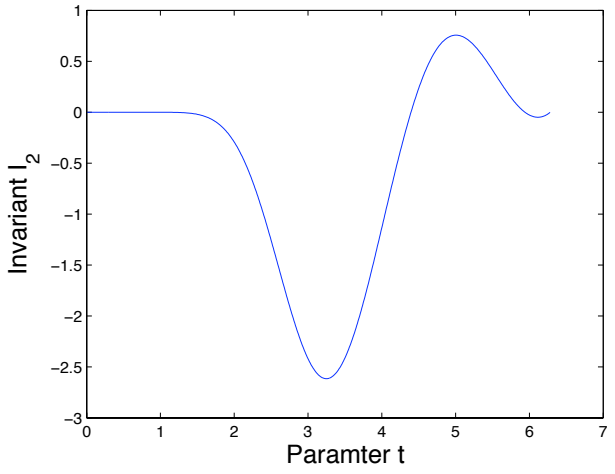

(b) Invariant $I_{2}$ for $\gamma(t)$ and $\overline{\gamma(t)}$

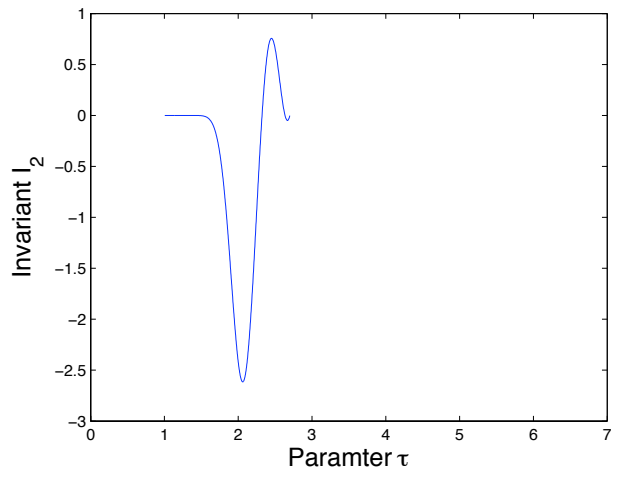

(d) Invariant $I_{2}$ for $\gamma(\tau)$ and $\overline{\gamma(\tau)}$

Figure 4: Dependence of invariants on re-prametrization: $\tau=\sqrt{t+1}$

of reflections and arbitrary scalings,

$$
\tilde{I}_{1}(t)=\frac{\left|I_{1}(t)\right|}{\max _{t}\left|I_{1}\right|}, \quad \tilde{I}_{2}(t)=\frac{\left|I_{2}\right|}{\max _{t}\left(I_{1}^{2}\right)} .
$$

Both invariants are reduced relative to the range of $\left|I_{1}\right|$. It is not difficult to show that $\max _{t}\left|I_{1}\right|=0$ on $\gamma$ if and only if $\gamma$ is a straight line. In this case the affine signature does not exist, but straight line regions can be easily detected by other means. The range of $\tilde{I}_{1}$ is from 0 to 1 . The full affine signature of a plane curve $\gamma(t)$ is obtained by, first, evaluating $\tilde{I}_{1}$ and $\tilde{I}_{2}$ on this curve and then by plotting the parameterized curve $\left(\tilde{I}_{1}(t), \tilde{I}_{2}(t)\right)$ in $\mathbb{R}^{2}$. For example, curves in Fig. 3-a and Fig. 3 .c are related by a non-area-preserving affine transformation. Their full affine signatures coincide as shown on Figure 6 .

\subsubsection{Global Affine Signatures for Curves in 3D}

To construct special affine signatures for spatial curve we use invariants $J_{1}$ and $J_{2}$ given by Eq. 22). Similarly to $2 \mathrm{D}$ case, the special affine signature of a spatial curve $\beta(t)$ is obtained by, first, evaluating $J_{1}$ and $J_{2}$ on this curve, and then plotting the parameterized curve $\left(J_{1}(t), J_{2}(t)\right)$ in $\mathbb{R}^{2}$. 


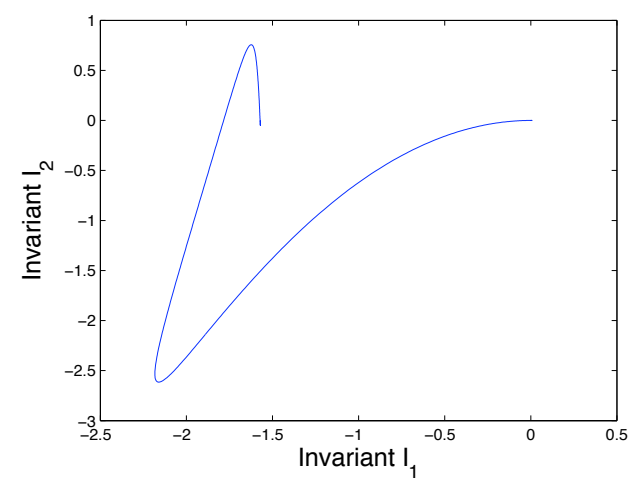

Figure 5: Signatures for $\gamma(t), \overline{\gamma(t)}, \gamma(\tau)$, and $\overline{\gamma(\tau)}$ coincide

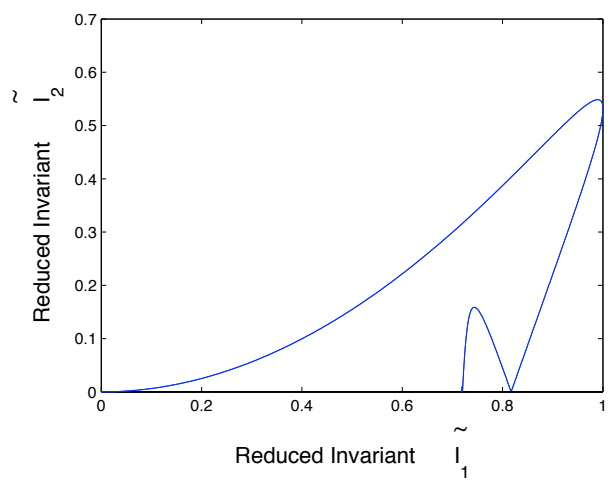

Figure 6: Full affine signatures for curves $\overline{\gamma(t)}$ and $\overline{\gamma_{a}(t)}$ coincide

For example, the signature of a spatial curve $\beta(t)=\left(\sin t-1 / 5 \cos ^{2} t+1 / 5,1 / 2 \sin t-\cos t+\right.$ $\left.1, \sin ^{2} t+\cos t-1\right)$, shown in Figure $7 \mathrm{f}$ a, is the plane curve shown on Fig 8 . A curve $\overline{\beta(t)}$ is obtained from $\beta$ by a special affine transformation $\left(\begin{array}{lll}0.3816 & 0.7631 & 1.1447 \\ 1.9079 & 1.5263 & 2.2894 \\ 2.6710 & 3.0526 & 3.4341\end{array}\right)$. A curve $\overline{\beta_{a}(t)}$ is obtained from $\beta$ by a full affine transformation $\left(\begin{array}{lll}1 & 2 & 3 \\ 4 & 5 & 6 \\ 9 & 8 & 7\end{array}\right)$. As Fig 8 illustrates, the special affine signatures of $\beta(t)$ and $\overline{\beta(t)}$ coincide.

Similar to the 2D case, the full affine signature for curves in $3 \mathrm{D}$ is obtained by reducing special affine invariants $J_{1}$ and $J_{2}$ by the range of $\left|J_{1}\right|$.

$$
\tilde{J}_{1}(t)=\frac{\left|J_{1}(t)\right|}{\max _{t}\left|J_{1}\right|}, \quad \tilde{J}_{2}(t)=\frac{J_{2}(t)}{\max _{t}\left(J_{1}^{2}\right)} .
$$

The full affine signature of a spatial curve $\beta(t)$ is obtained by first evaluating $\tilde{J}_{1}$ and $\tilde{J}_{2}$ on this curve, and by then plotting the parameterized curve $\left(\tilde{J}_{1}(t), \tilde{J}_{2}(t)\right)$ in $\mathbb{R}^{2}$. The full affine 
Figure 7:

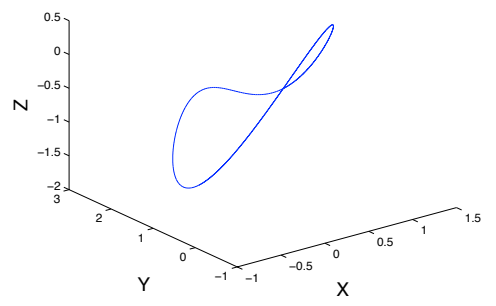

(a) original curve $\beta(t)$

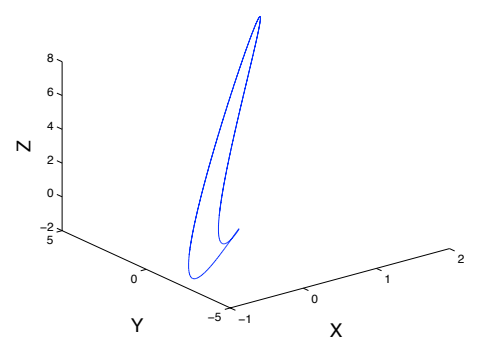

(b) special affine transformation $\overline{\beta(t)}$

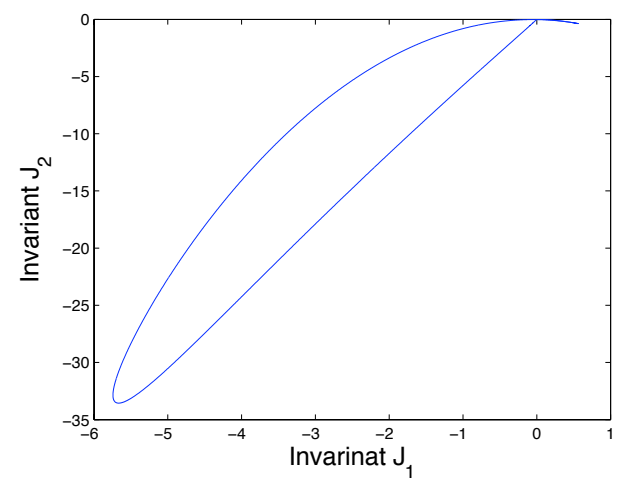

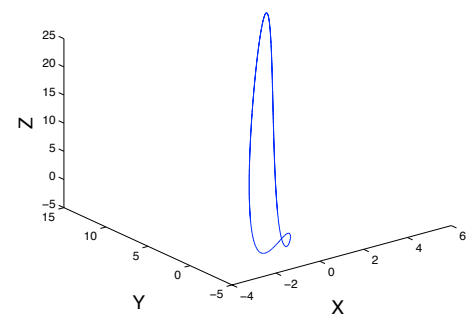

(c) full affine transformation $\overline{\beta_{a}(t)}$

Figure 8: Signatures for $\beta(t)$ and $\overline{\beta(t)}$ coincide.

signatures of $\beta, \bar{\beta}$ and $\bar{\beta}_{a}$ coincide as shown in Fig 9 .

The advantage of global signatures is their independence of parametrization, whereas the result of evaluation of invariants $J_{1}$ and $J_{2}$ on a curve depends on the choice of parametrizations similarly to $I_{1}$ and $I_{2}$ in $2 \mathrm{D}$ case. The disadvantage of global signatures is in their dependence on the choice of the initial point of a curve. The local signature construction in the next section overcomes this dependence.

\subsection{Local Integral Affine Signatures}

The signatures defined in the previous section can not be used for the classification unless the initial point of a curve is known. This becomes an obstacle for comparing closed curves or for matching parts of contours. For illustration, let us choose two different initial points, black circle or red star, on the planar curve in Figure 10. The resulting global affine signatures are different as illustrated in Figures $11 \mathrm{ra}$ and Figures $11 \mathrm{fb}$. We overcome the dependence on the initial point by introducing local signatures. To proceed with the construction of the local signature, we replace the integration from the initial point with integration on local segments. To retain affine invariant properties of the signatures, we need to partition the curve in an invariant manner. Such partition can be achieved using the notion of affine arc-length from classical differential geometry. Our goal, 


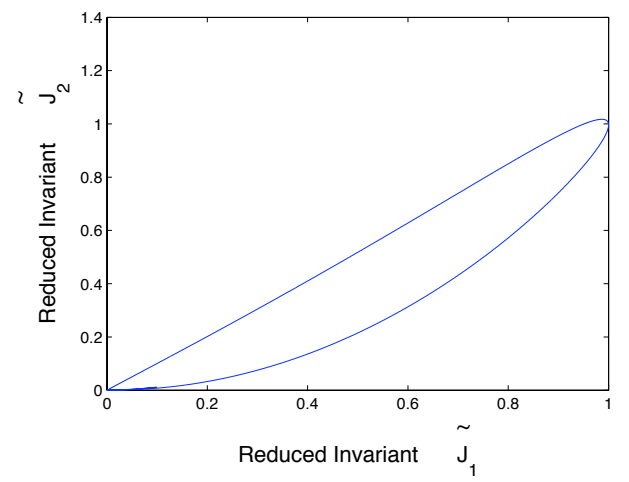

Figure 9: Full affine signatures for $\beta(t), \overline{\beta(t)}, \overline{\beta_{a}(t)}$

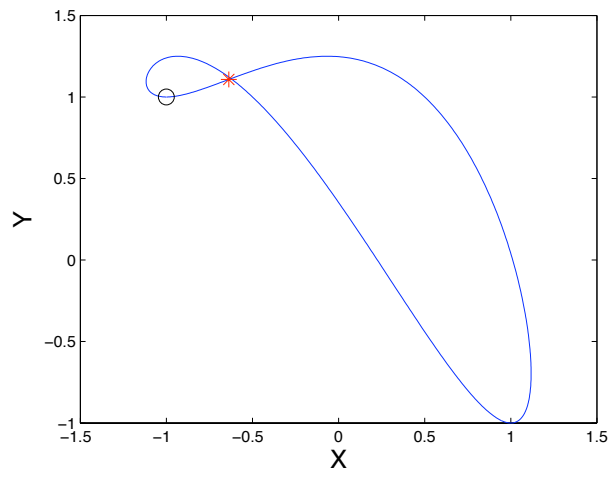

Figure 10: A planar curve with two different choices of the initial points

however, is to propose a derivative free method, and so we use the lowest order integral invariants, namely $I_{1}$ for plane curves and $J_{1}$ for spatial curve to obtain an equi-affine partition of a given curve. The details are described in the following subsections.

\subsubsection{Local Affine Signatures for Curves in 2D}

We will use $I_{1}$ to partition a given curve into equi-affine sub-segments. Assume that $\gamma$ is parametrized by $t \in[0,1]$. For this purpose we define an evaluation of invariants on sub-segments of $\gamma$. Recall that the integration in the integral variables is performed from the initial point $\gamma(0)$ to a current point on the curve $\gamma(t)$. For instance, $I_{1}(t)=\int_{0}^{t} X d Y-\frac{1}{2} X Y$, where $X=x(t)-x(0)$ and $Y=y(t)-y(0)$. Thus $I_{1}(t)$ is a function from $[0,1]$ to $\mathbb{R}$.

We define the evaluation of an invariant on sub-segments of $\gamma$ by treating the starting point of a segment as the initial point, and computing the value of integral variables at the end point. In particular, for a sub-segment defined by the parameter range $[p, q] \subset[0,1]$, we may compute the localization $I_{1}^{[p, q]}=\int_{p}^{q}(x(t)-x(p)) d y(t)-\frac{1}{2}(x(q)-x(p))(y(q)-y(p))$, and similarly for invariants $I_{2}$ and $I_{3}$ defined by Eq.(13). We note that the evaluation of an invariant on a segment 


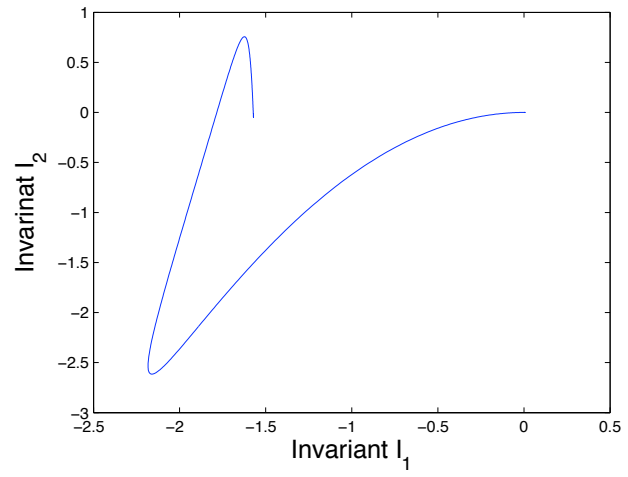

(a) Initial point is the black circle in Fig, 10

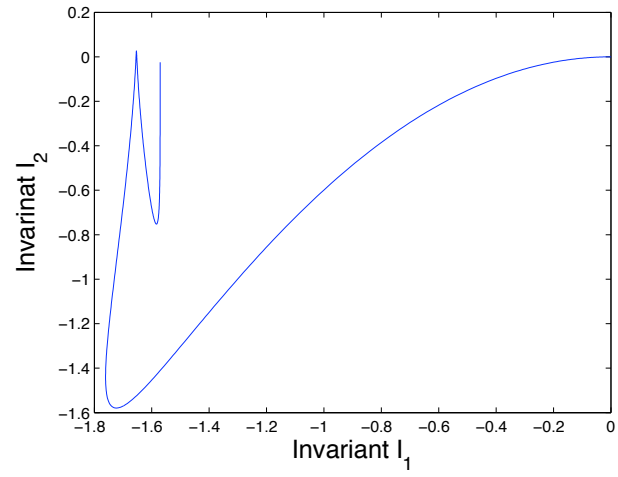

(b) Initial point is the red star in Fig 10

Figure 11: Global signatures for the same curve with two different choices of the initial point

is a real number.

We choose a sufficiently small $\Delta>0$ and define an equi-affine partition $0=t_{0}<t_{1}<\cdots<$ $t_{N}=1$ of the curve $\gamma(t), t \in[0,1]$ into sub-segments by the condition

$$
\left|I_{1}^{\left[t_{i-1}, t_{i}\right]}\right|=\Delta .
$$

In practice we choose $\Delta$ proportionally to the maximum of the absolute value of $I_{1}$, i.e., we choose an integer $M$ and set $\Delta=\frac{\max _{t}\left|I_{t}\right|}{M}$. Note that the total number $N$ of segments that we obtain in general differs from $M$. The local discrete special affine signature of $\gamma$ is defined by an evaluation of $I_{2}$ and $I_{3}$ on the intervals $\left[t_{i-1}, t_{i}\right], i=1 . . N$, that is a set of points with coordinates $\left(I_{2}^{\left[t_{i-1}, t_{i}\right]}, I_{3}^{\left[t_{i-1}, t_{i}\right]}\right) i=1 . . N$. Figure 12 illustrates that the local discrete special affine signature

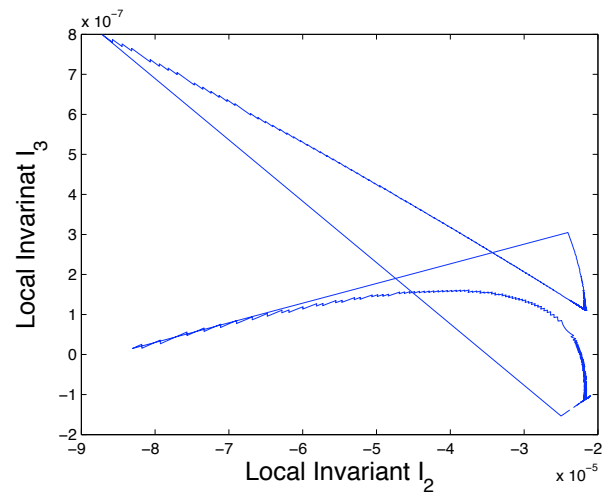

Figure 12: Local special affine signature for the curve shown on Figure 10

for the curve shown in Fig 10, with different starting points coincides. To obtain local discrete affine signature of $\gamma$, we use reduced invariants, that is we plot $\left(\frac{I_{2}^{\left[t_{i-1}, t_{i}\right]}}{\max _{t}\left(I_{1}^{2}\right)}, \frac{I_{3}^{\left[t_{i-1}, t_{i}\right]}}{\max _{t}\left|I_{1}^{3}\right|}\right), i=1 . . N$. 


\subsubsection{Local Affine Signatures for Curves in 3D}

For a spatial curve we proceed in a similar manner as for plane curves. We use invariant $J_{1}$ to partition a curve $\gamma(t), t \in[0,1]$ into $N$ sub-intervals defined by $a=t_{0}<t_{1}<\cdots<t_{N}=b$ such that $J_{1}^{\left[t_{i-1}, t_{i}\right]}=\Delta, i=1 . . N$, where $\Delta>0$ is proportional to the maximum of the absolute value of $J_{1}$. We define a local special affine signature by evaluation of $J_{2}$ and $J_{3}$ on the intervals $\left[t_{i-1}, t_{i}\right], i=1 . . N$, that is by a set of points on the plane with coordinates $\left(J_{2}^{\left[t_{i-1}, t_{i}\right]}, J_{3}^{\left[t_{i-1}, t_{i}\right]}\right) i=$ 1..N.

Figure 13 -b shows the local special affine signature for a curve shown on Figure 13 a. The signature does not depend on our choice of initial point.

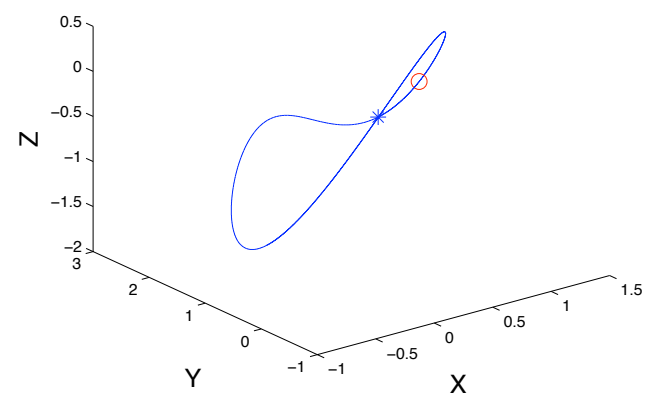

(a) A curve with two different choices of an initial point

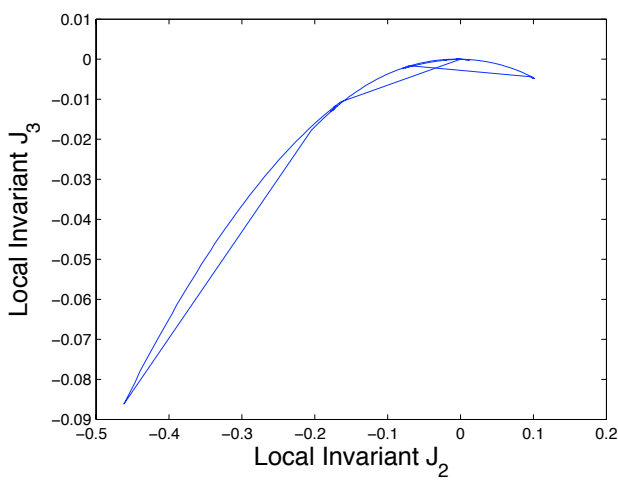

(b) Local special affine signature

Figure 13: A curve and its local special affine signature

\section{3D Object Classification}

The features of many computer vision and pattern analysis problems are spatial curves. We can hence view the classification problem as that of assorting the similarity of curves in 3D, in particular, when subjected to affine transformations. In this section, we apply integral special affine invariants $J_{1}$ and $J_{2}$, the global special affine signature, and the local special affine signature to classify curves in 3D under special affine transformations. The performance of each of the proposed methods is evaluated. Applying these invariants to classification of 3D objects based on a set of characteristic spatial curves is in line of [1], and will be considered in subsequent publications.

\subsection{Experimental Design}

The Princeton Shape Benchmark [31] provides a repository of 3D models. A subset of three models are shown in Figure 14. We extract a total of 100 characteristic curves, and each of them are re-sampled to 5000 points with the same arch-length. We applied to each curve 9 randomly generated 3D special affine transformations as shown on (Figure 15). To make this problem even more challenging and to illustrate the noise sensitivity of the proposed approach, gaussian noise 

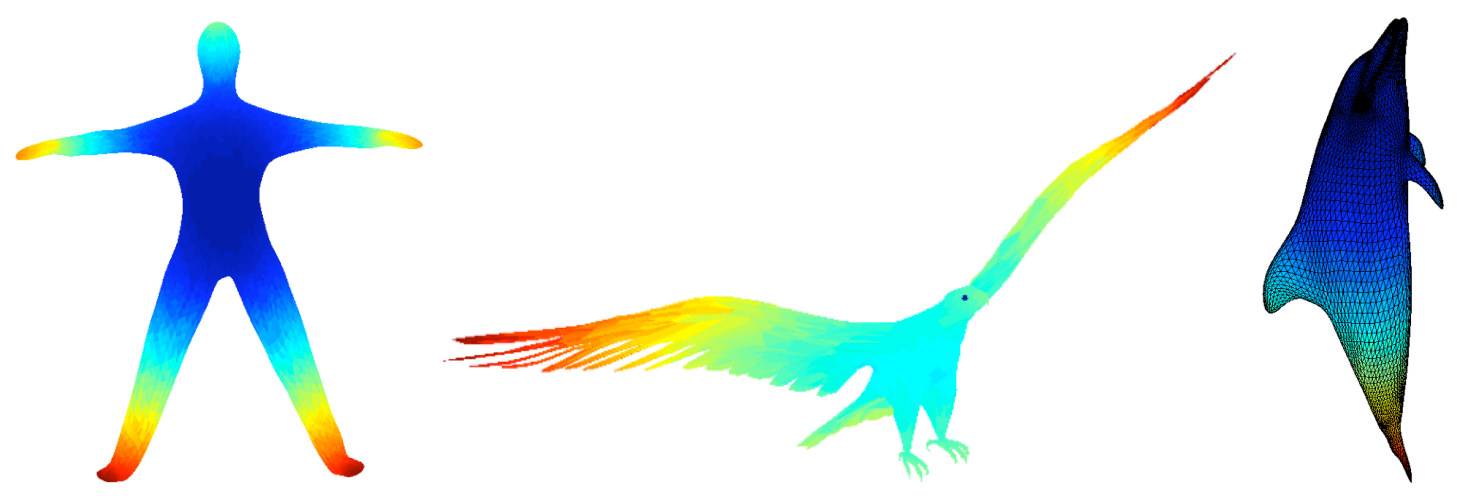

Figure 14: 3D models from The Princeton Shape Benchmark

with distribution $N\left(0, \sigma^{2}\right)$ is added to each of the variations. We therefore obtain a classification set of 900 curves that has to be separated into 100 equivalent classes under affine transformations. The training set consists of 100 original curves without any noise and transformation. The discrimination power and sensitivity to noise are analyzed using the error rate of classification. We implemented a Nearest Neighbor (NN) Classifier in a Euclidean Space using Euclidean distance. In order to illustrate the advantages of the signature, we design two experiments. The first experiment uses a common parametrization for both the training and testing, while in the second experiment, we choose two different parametrizations (samplings) for the testing data.
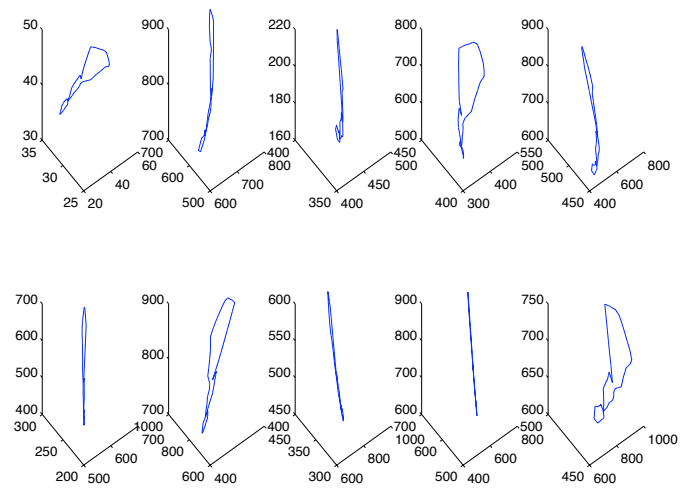

Figure 15: A curve and 9 variations of it under affine transformation

\section{$5.2 \quad$ Experimental Results}

Three experiments are carried out with different noise variance, namely $\sigma=2$ (Fig. 16), $\sigma=1$, and $\sigma=0.5$. The error rates of the three different sigma settings with the same parametrization are shown in Table.I.

In Table I, both the integral invariants and the signatures perform well as indicated by the error rates. For comparison, the classical differential invariants have a classification error rate more then $80 \%$, which makes the differential invariants practically useless. Since the order of 


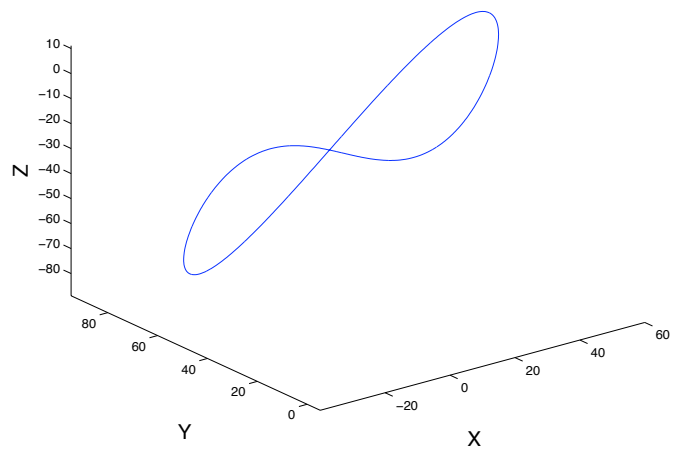

(a)

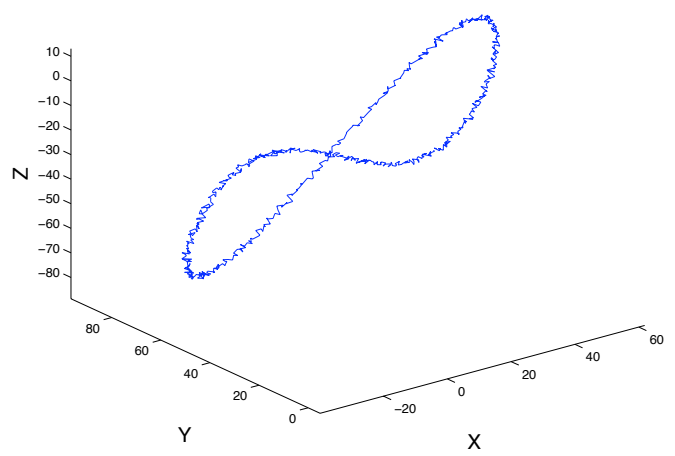

(b)

Figure 16: (a) A spatial curve without noise (b) with Gaussian Noise $N(0,4)$,

Table 1: Classification error rate with same parametrization, same initial points

\begin{tabular}{|c|c|c|c|c|}
\hline Noise variance & $J_{1}$ & $J_{2}$ & Global signature & Local signature \\
\hline$\sigma=0.5$ & $\mathbf{0 . 0 0 2 2}$ & 0.0472 & 0.06 & 0.07 \\
$\sigma=1$ & $\mathbf{0 . 0 4}$ & 0.12 & 0.15 & 0.17 \\
$\sigma=2$ & $\mathbf{0 . 0 7 8 9}$ & 0.2233 & 0.28 & 0.32 \\
\hline
\end{tabular}

Table 2: Classification error rate with different parametrization, same initial points

\begin{tabular}{|c|c|c|c|c|}
\hline Noise variance & $J_{1}$ & $J_{2}$ & Global signature & Local signature \\
\hline$\sigma=0.5$ & 0.42 & 0.61 & $\mathbf{0 . 0 6}$ & $\mathbf{0 . 0 7}$ \\
$\sigma=1$ & 0.48 & 0.70 & $\mathbf{0 . 1 5}$ & $\mathbf{0 . 1 7}$ \\
$\sigma=2$ & 0.56 & 0.83 & $\mathbf{0 . 2 8}$ & $\mathbf{0 . 3 2}$ \\
\hline
\end{tabular}

Table 3: Classification error rate with different parametrization, different initial points

\begin{tabular}{|c|c|c|c|c|}
\hline Noise variance & $J_{1}$ & $J_{2}$ & Global signature & Local signature \\
\hline$\sigma=0.5$ & 0.87 & 0.95 & 0.95 & $\mathbf{0 . 0 7}$ \\
$\sigma=1$ & 0.91 & 0.97 & 0.97 & $\mathbf{0 . 1 7}$ \\
$\sigma=2$ & 0.94 & 0.98 & 0.98 & $\mathbf{0 . 3 2}$ \\
\hline
\end{tabular}


integral variables involved in $J_{2}$ is higher than $J_{1}$, as well as the explicit form of $J_{2}$ is more complicated than $J_{1}$, the performance of $J_{2}$ is not as good as that of $J_{1}$. The global signature is constructed with both $J_{1}$ and $J_{2}$, and the local signature is based on $J_{2}$ and $J_{3}$. The performance of these signatures is therefore slightly worse than $J_{2}$.

If the parameterizations are not the same, the plots of invariants $J_{1}$ and $J_{2}$ with respect to a parameter can not be used for a classification purpose as illustrated in table II. Even with the lowest noise variance, the error rates are more than 0.4 for $J_{1}$ and 0.6 for $J_{2}$. However, neither the global signature nor local signature are affected.

If we make an arbitrary selection of the initial points, both individual invariants $\left(J_{1}\right.$ and $\left.J_{2}\right)$ and global signature have poor performance as shown in Table III. Only local signature may be used to characterize a curve.

As a conclusion, if the training data and testing data have similar parameterization and same initial point, either invariants or signatures may be used. Under different parametrization, the global signature is the best choice. With an unknown starting point, the local signature is the only solution.

\section{CONCLUSIONS}

In this paper, we presented explicit formulas for affine integral invariants for plane and space curves in terms of Euclidean invariants. Based on the invariants, we constructed signatures for special affine groups and full affine groups for these curves. Although we have focused here on a more complex case of affine transformations, the integral Euclidean invariants, presented here, can be used to classify curves under Euclidean transformations [10.

Integral invariants are functions of the parameter, and hence depend on the parameterization. Global integral signatures provide a classification method independent of parameterization (curve sampling). A global integral signature depends on the choice of the initial point. Local integral signatures provide a classification method independent of the choice of the initial point, they can hence be used on images with occlusions and for comparing fragments of contours. They are slightly more sensitive to noise than global signatures.

As an experiment, a classification of characteristic curves of 3D objects, subjected to random affine transformation and noise, was conducted by using individual invariants and global and local signatures. Integral invariants allow us to perform noise tolerant classification method for curves with respect to the affine transformations (for comparison: differential invariants give $80 \%$ error rate).

\section{APPENDIX: derivation of invariants}

\subsection{Cross-section and moving frame map}

Building on the works [12, 16, 11, Fels and Olver [7] generalized Cartan's normalization procedure [4], and proposed a general algorithm for computing invariants. The Fels-Olver algorithm relies on a map $\rho: S \rightarrow G$ with an equivariant property:

$$
\rho(g \cdot s)=\rho(s) \cdot g^{-1}, \forall g \in G, \forall s \in S .
$$


From Theorem 4.4 in [7, it follows that such map exists if and only if the action of $G$ is free and, in addition, there exists a global cross-section, i.e a subset $\mathcal{K} \subset S$ that intersects each orbit $O_{s}$ at a unique point. Indeed, under the above assumption the map $\rho$ may be defined by the condition $\rho(s) \cdot s \in \mathcal{K}$. Then $\rho(s) \cdot s=\rho(g \cdot s) \cdot(g \cdot s)$ is the unique point of the intersection of $O_{s}$ and $\mathcal{K}$. From the freeness it follows that $s$ may be "cancelled" and hence the condition $(26)$ is satisfied.

If $G$ is a Lie group acting smoothly on $\mathbb{R}^{n}$ and both $S \subset \mathbb{R}^{n}$ and $\mathcal{K} \subset S$ are smooth submanifolds, then $\mathbb{R}^{n}$-coordinate components of the projection $\iota(s)=\rho(s) \cdot s: S \rightarrow \mathcal{K}$ are smooth invariant functions, called normalized invariants. Normalized invariants contain a maximal set of functionally independent invariants, and have a replacement property, which allows us to rewrite any invariant in terms of them by simple substitution [7, 15, 14].

Although, a global smooth cross-section does not always exist, a local smooth cross-section 4 passing through every point of $S$ may be found for every semi-regular action. $5^{5}$ The freeness assumption can be also relaxed to a semi-regularity assumption. With these weaker assumptions the above method can be used to construct local invariants [7, 14] $]^{6}$

For algebraic groups acting on algebraic varieties, a purely algebraic counterpart of the FelsOlver construction was obtained in [15, 14. The algebraic method can be combined with the inductive approach described below. In some particular examples, including the 3D example presented here, the computation based on the moving frame map $\rho$ turns out to be more practical.

When the group $G$ is of relatively large dimension, computation of invariants by either a geometric or algebraic approach becomes challenging. In [17] two modifications of the moving frame method were proposed to simplify the computation by splitting it into two steps: invariants of a subgroup $A \subset G$ are first computed, and then invariants of the entire group are constructed in terms of those. For the problem at hand, we use one of these modifications, called the inductive approach, which is applicable when a group factors into a product of two subgroups.

\subsection{Inductive approach}

Definition 7.1 A group $G$ factors as a product of its subgroups $A$ and $B$ if for any $g \in G$ there are $a \in A$ and $b \in B$ such that $g=a b$.

We write $G=A \cdot B$. If in addition $A \cap B=e$, then for each $g \in G$ there are unique elements $a \in A$ and $b \in B$ such that $g=a b$.

Theorem $G=B \cdot A$, such that $A \cap B$ is discrete, and $G$ acts freely on a manifold $S$ then $\forall s \in S$ there exists a local cross-section $\mathcal{K}_{A}$, containing $s$, invariant under the action of the subgroup $B$. From Lemma 4.7 in [17] it follows that invariants of $G$ can be constructed from the invariants of $A$ using the following method.

\section{Inductive method:}

1. Restrict the $G$-action to $A$. Find a local cross-section $\mathcal{K}_{A} \subset S$ for the action of $A$ which is invariant under the action of $B$.

\footnotetext{
${ }^{4}$ A local cross-section is defined on an open subset of $U \subset S$ and $\forall s \in U$ intersects each connected component of $O_{s} \cap U$ at a unique point.

${ }^{5}$ An action of $G$ is called semi-regular if all orbits have the same dimension.

${ }^{6}$ A function $f$, defined on an open subset $U$ of $S$, is a local invariant if $\forall s \in U$ there exists an open neighborhood $G_{s}$ of $e \in G$ s.t. condition (1) is satisfied for all $g \in G_{s}$.
} 
2. Construct a moving frame map $\rho_{A}: S \rightarrow A$ defined by the condition $\rho_{A}(s) \cdot s \in \mathcal{K}_{A}, \forall s \in$ $S$, by solving the corresponding equations. Composition of coordinate functions with the projection $\iota(s)=\rho_{A}(s) \cdot s: S \rightarrow \mathcal{K}_{A}$ are invariant with respect to the action of $A$.

3. Restrict the action of $G$ to the action of its subgroup $B$ on the invariant subset $\mathcal{K}_{A}$ and choose a local cross-section $\mathcal{K}_{B} \subset \mathcal{K}_{A}$.

4. Construct a moving frame map $\rho_{B}: \mathcal{K}_{A} \rightarrow B$ defined by the condition $\rho_{B}(s) \cdot z \in \mathcal{K}_{B}, \forall z \in$ $\mathcal{K}_{A}$, by solving the corresponding equations.

5. The $G$-moving frame map $\rho: S \rightarrow G$ is defined by $\rho(s)=\rho_{B}\left(\rho_{A}(s) \cdot s\right) \rho_{A}$, and $G$-invariants are the coordinate components of $\rho(s) \cdot s=\rho_{B}\left(\rho_{A}(s) \cdot s\right) \cdot\left(\rho_{A}(s) \cdot s\right)=\rho_{B}\left(\iota_{A}(s)\right) \cdot \iota_{A}(s)$.

\subsection{Integral affine invariants for curves in $2 \mathrm{D}$}

We have a product decomposition $S L(2)=B \cdot A$, where $B=\left\{\left(\begin{array}{cc}b_{11} & b_{12} \\ 0 & \frac{1}{b_{11}}\end{array}\right) \mid b_{11}>0\right\}$ and $A=S O(2)$ is a group of rotations. The intersection $B \cap A=\{e\}$, and therefore we can apply the inductive method as follows.

1. We restrict the $S L(2)$-action (11) to the subgroup $\mathcal{S O}(2)$ of the rotation matrices by setting $a_{11}=\cos \phi, a_{12}=-\sin \phi, a_{21}=\sin \phi, a_{22}=\cos \phi$. A subset $\mathcal{K}_{A}$ defined by conditions, $Y=0, X>0$ serves as a cross-section on an open subset of the integral jet bundle. Moreover $\mathcal{K}_{A}$ is invariant under the restriction of (11) to subgroup $B$.

2. The corresponding moving frame map $\rho_{A}(s)=\left(\begin{array}{cc}\frac{X}{\sqrt{X^{2}+Y^{2}}} & \frac{Y}{\sqrt{X^{2}+Y^{2}}} \\ -\frac{Y}{\sqrt{X^{2}+Y^{2}}} & \frac{X}{\sqrt{X^{2}+Y^{2}}}\end{array}\right)$ is obtained by solving the equation $\bar{Y}=0$ with the condition $\bar{X}>0$ (see $\sqrt{12}$ )). The projection $\iota_{A}: \mathbb{R}^{5} \rightarrow$ $\mathcal{K}_{A}$, obtained by substitution $\rho_{A}$ into $(11)$, produces a point whose coordinates are invariant under the action of $\mathcal{S} O(2)$. Non-constant normalized invariants are given by $(12)$ and $Y_{\mathcal{S E}}=0$ is the remaining constant invariant.

3. We now restrict the action (11) to the action of a subgroup $B$ on an invariant subset $\mathcal{K}_{A}$. We obtain the following transformations.

$$
\begin{aligned}
& \overline{X_{A}}=b_{11} X_{A} \text {, } \\
& \overline{Y_{A}^{[1,1]}}=\frac{1}{b_{11}} Y_{A}^{[1,1]} \text {, } \\
& \overline{Y_{A}^{[1,2]}}=\frac{1}{b_{11}^{2}} Y_{A}^{[1,2]} \text {, } \\
& \overline{Y_{A}^{[3,0]}}=b_{11}^{2} Y_{A}^{[3,0]}+3 b_{12}^{2} Y_{A}^{[1,2]}+3 b_{11} b_{12} Y_{A}^{[2,1]} \text {. } \\
& \begin{array}{l}
\overline{Y_{A}^{[1,0]}}=Y_{A}^{[1,0]} \\
\overline{Y_{A}^{[2,0]}}=b_{11} Y_{A}^{[2,0]}+2 b_{12} Y_{A}^{[1,1]} \\
\overline{Y_{A}^{[2,1]}}=Y_{A}^{[2,1]}+2 \frac{b_{12}}{b_{11}} Y_{A}^{[1,2]},
\end{array}
\end{aligned}
$$

A subset $\mathcal{K}_{B} \subset \mathcal{K}_{A}$ defined by the equations $X_{A}=1, \quad Y_{A}^{[2,0]}=0$ serves as a cross-section on the subset of $\mathcal{K}_{A}$, where $Y_{A}^{[1,1]} \neq 0$. 
4. This leads to the moving frame map $\rho_{B}(s)=\left(\begin{array}{cc}\frac{1}{X_{A}} & -\frac{Y_{A}^{[2,0]}}{2 Y_{A}^{[1,1]} X_{A}} \\ 0 & X_{A}\end{array}\right)$. The projection $\iota_{B}: \mathcal{K}_{A} \rightarrow$ $\mathcal{K}_{B}$, defined by $\iota_{B}(s)=\rho_{B}(s) \cdot s$, produces a point with coordinates

$$
\begin{array}{ll}
X_{B}=1, & Y_{B}^{[1,0]}=Y_{A}^{[1,0]}, \\
Y_{B}^{[1,1]}=X_{A} Y_{A}^{[1,1]}, & Y_{B}^{[2,0]}=0, \\
Y_{B}^{[1,2]}=X_{A}^{2} Y_{A}^{[1,2]}, & Y_{B}^{[2,1]}=Y_{A}^{[2,1]}-\frac{Y_{A}^{[2,0]}}{Y_{A}^{[1,1]}} Y_{A}^{[1,2]}, \\
Y_{B}^{[3,1]}=\frac{1}{X_{A}^{2}}\left(Y_{A}^{[3,0]}+\frac{3}{2} \frac{Y_{A}^{[2,0]}}{Y_{A}^{[1,1]}} Y_{A}^{[2,1]}+\frac{3}{4}\left(\frac{Y_{A}^{[2,0]}}{Y_{A}^{[1,1]}}\right)^{2} Y_{A}^{[1,2]}\right) . &
\end{array}
$$

invariant under the $B$-action 27 on $\mathcal{K}_{A}$.

5. Replacing coordinates $Y_{A}^{[i, j]}$ with the corresponding $Y_{\mathcal{S} E}^{[i, j]}$ given by 12 produces independent invariants (13).

\subsection{Affine integral invariants for curves in $3 \mathrm{D}$}

We have a product decomposition $S L(3)=B \cdot A$, where $B=\left\{\left(\begin{array}{ccc}b_{11} & b_{12} & b_{13} \\ 0 & b_{22} & b_{23} \\ 0 & 0 & \frac{1}{b_{11} b_{22}}\end{array}\right) \mid b_{11}>0, b_{22} \neq 0\right\}$ and $A=S O(3)$ is a group of rotations. The intersection $B \cap A=\{e\}$ is trivial. We again follow the steps of the inductive method.

1. We restrict the $\mathcal{S} L(3)$ to the action of $\mathcal{S O}(3)$ whose elements can be represented as the product of three rotations:

$$
\left(\begin{array}{ccc}
1 & 0 & 0 \\
0 & \cos \psi & -\sin \psi \\
0 & \sin \psi & \cos \psi
\end{array}\right)\left(\begin{array}{ccc}
\cos \phi & 0 & \sin \phi \\
0 & 1 & 0 \\
-\sin \phi & 0 & \cos \phi
\end{array}\right)\left(\begin{array}{ccc}
\cos \theta & -\sin \theta & 0 \\
\sin \theta & \cos \theta & 0 \\
0 & 0 & 1
\end{array}\right)
$$

A subset $\mathcal{K}_{A}$, defined by conditions, $Y=0, Z=0, Z_{011}=0, X>0$ serves as a cross-section on the open subset of the integral jet bundle where $X^{2}+Y^{2}+Z^{2}>0$. The cross-section $\mathcal{K}_{A}$ is invariant under the action of $B$.

2. The corresponding moving frame map $\rho_{A}$ is obtained by solving the equation $\bar{Y}=0, \bar{Z}=$

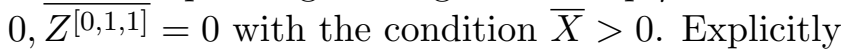

$$
\begin{aligned}
& \cos \theta=\frac{X}{\sqrt{X^{2}+Y^{2}}}, \quad \cos \phi=\frac{\sqrt{X^{2}+Y^{2}}}{\sqrt{X^{2}+Y^{2}+Z^{2}}}, \quad \cos \psi=\frac{Z_{R}^{[0,2,0]}}{\sqrt{\left(Z_{R}^{[0,2,0]}\right)^{2}+4\left(Z_{R}^{[0,1,1]}\right)^{2}}}, \\
& \sin \theta=-\frac{Y}{\sqrt{X^{2}+Y^{2}}}, \quad \sin \phi=\frac{Z}{\sqrt{X^{2}+Y^{2}+Z^{2}}}, \quad \sin \psi=-2 \frac{Z_{R}^{[0,1,1]}}{\sqrt{\left(Z_{R}^{[0,2,0]}\right)^{2}+4\left(Z_{R}^{[0,1,1]}\right)^{2}}} .
\end{aligned}
$$

we $Z_{R}^{[0,1,1]}$ and $Z_{R}^{[0,2,0]}$ are given on the last page of the Appendix. The corresponding set of $\mathcal{S O}(3)$ invariants is given by 20 . 
3. We now restrict the action $\mathcal{S} L(3)$-action to the action of a subgroup $B$ on an invariant subset of $\mathcal{K}_{A}$. We obtain the following transformations:

$$
\begin{aligned}
\overline{X_{A}} & =b_{11} X_{A}, \\
\overline{Z_{A}^{[0,1,0]}} & =\frac{1}{b_{11}} Z_{A}^{[0,1,0]} \\
\overline{Z_{A}^{[1,0,0]}} & =\frac{1}{b_{22}} Z_{A}^{[1,0,0]}+\frac{b_{12}}{b_{11} b_{22}} Z_{A}^{[0,1,0]} \\
\overline{Y_{A}^{[1,0,0]}} & =b_{11} b_{22} Y^{[1,0,0]}-b_{13} b_{22} Z_{A}^{[0,1,0]}+b_{11} b_{23} Z_{A}^{[1,0,0]}+b_{12} b_{23} Z_{A}^{[0,1,0]} \\
\overline{Z_{A}^{[0,2,0]}} & =\frac{b_{22}}{b_{11}} Z_{A}^{[0,2,0]} \\
\overline{Z_{A}^{[1,0,1]}} & =\frac{1}{b_{11} b_{22}^{2}} Z_{A}^{[1,0,1]} \\
\overline{Z_{A}^{[1,1,0]}} & =Z_{A}^{[1,1,0]}+\frac{b_{23}}{b_{22}} Z_{A}^{[1,0,1]}+\frac{b_{12}}{b_{11}} Z_{A}^{[0,2,0]} \\
\overline{Y_{A}^{[1,0,1]}} & =Y_{A}^{[1,0,1]}+\frac{b_{23}}{b_{22}} Z_{A}^{[1,0,1]}-\frac{b_{12}}{2 b_{11}} Z_{A}^{[0,2,0]}
\end{aligned}
$$

A subset $\mathcal{K}_{B} \subset \mathcal{K}_{A}$ defined by equations

$$
Z_{A}^{[0,1,0]}=1, Z_{A}^{[1,0,0]}=1, Y_{A}^{[1,0,0]}=1, Z_{A}^{[0,2,0]}=1, Z_{A}^{[1,1,0]}=1
$$

is a cross-section on an open subset of $\mathcal{K}_{A}$.

4. The corresponding moving frame map $\rho_{B}$ is

$$
\begin{aligned}
b_{11} & =Z_{A}^{[0,1,0]}, \quad b_{12}=-\frac{Z_{A}^{[0,2,0]} Z_{A}^{[1,0,0]}-Z_{A}^{[0,1,0]}}{Z_{A}^{[0,2,0]}}, \\
b_{22} & =\frac{Z_{A}^{[0,1,0]}}{Z_{A}^{[0,2,0]}}, \quad b_{23}=\frac{-Z_{A}^{[0,1,0]} Z_{A}^{[1,1,0]}+Z_{A}^{[0,2,0]} Z_{A}^{[1,0,0]}}{Z_{A}^{[0,2,0]} Z_{A}^{[1,0,1]}}, \\
b_{13} & =-\frac{-Z_{A}^{[0,1,0]^{2}} Z_{A}^{[0,2,0]} Z_{A}^{[1,0,0]}+Z_{A}^{[0,1,0]^{3}} Z_{A}^{[1,1,0]}-Z_{A}^{[0,2,0]} Z_{A}^{[1,0,1]} Z_{A}^{[0,1,0]^{2}} Y_{A}^{[1,0,0]}+Z_{A}^{[1,0,1]} Z_{A}^{[0,2,0]}{ }^{2}}{Z_{A}^{[0,2,0]} Z_{A}^{[1,0,1]} Z_{A}^{[0,1,0]}{ }^{2}} .
\end{aligned}
$$

The coordinate components of the projection $\rho_{B}(s) \cdot s: \mathcal{K}_{A} \rightarrow \mathcal{K}_{B}$

$$
\begin{aligned}
X_{B} & =Z_{A}^{[0,1,0]} X_{A} \\
Z_{B}^{[1,0,1]} & =\frac{Z_{A}^{[1,0,1]} Z_{A}^{[0,2,0]^{2}}}{Z_{A}^{[0,1,0]^{3}}}, \\
Y_{B}^{[1,0,1]} & =\frac{2 Y_{A}^{[1,0,1]} Z_{A}^{[0,1,0]}-2 Z_{A}^{[0,1,0]} Z_{A}^{[1,1,0]}+3 Z_{A}^{[0,2,0]} Z_{A}^{[1,0,0]}}{2 Z_{A}^{[0,1,0]}}-\frac{1}{2} .
\end{aligned}
$$

are invariant under the action of $B$ on $\mathcal{K}_{A}$. 
5. Replacing coordinates $Y_{A}^{[i, j, k]}, Z_{A}^{[i, j, k]}$ with the corresponding $Y_{\mathcal{S} E}^{[i, j, k]}, Z_{\mathcal{S} E}^{[i, j, k]}$ given by 20 produces independent invariants (21). 
The following auxiliary expressions were used in the paper where $r=\sqrt{X^{2}+Y^{2}}, R=\sqrt{X^{2}+Y^{2}+Z^{2}}$ :

$$
\begin{aligned}
& Z_{R}^{[0,1,0]}=-\frac{1}{2 R}\left(X Y Z-2 X Z^{[0,1,0]}+2 Y Z^{[1,0,0]}-2 Z Y^{[1,0,0]}\right) \\
& Z_{R}^{[1,0,0]}=-\frac{1}{2 r}\left(X^{2} Z-2 X Z^{[1,0,0]}+Y^{2} Z-2 Y Z^{[0,1,0]}\right) \\
& Y_{R}^{[1,0,0]}=-\frac{1}{2 r R}\left(Y X^{3}+X Y^{3}-2 Y^{2} Y^{[1,0,0]}-2 Z Y Z^{[1,0,0]}-2 X^{2} Y^{[1,0,0]}+2 Z X Z^{[0,1,0]}\right) \\
& Z_{R}^{[0,1,1]}=-\frac{1}{6 r R}\left(2 Y X^{3} Z^{2}-6 X^{3} Z^{[0,1,1]}+6 X^{2} Y Z^{[1,0,1]}-6 X^{2} Z Y^{[1,0,1]}\right. \\
& -6 X Z^{[0,1,1]} Y^{2}+3 X Y Z Z^{[0,2,0]}+4 Z^{2} Y^{3} X+6 X Y Z X^{[1,0,1]}-6 X Z^{2} X^{[1,1,0]}-6 Y^{2} Z Z^{[1,1,0]} \\
& \left.-6 Y^{2} Z Y^{[1,0,1]}+6 Y^{3} Z^{[1,0,1]}-3 Z^{2} Y X^{[0,2,0]}\right) \\
& Z_{R}^{[0,2,0]}=-\frac{1}{3 r R}\left(-3 X^{2} Z^{[0,2,0]}-2 Y^{2} X^{2} Z+6 X Y Z^{[1,1,0]}+3 X Z X^{[0,2,0]}+6 Y^{2} X^{[1,0,1]}-6 Z Y X^{[1,1,0]}\right) \\
& Z_{R}^{[1,0,1]}=\frac{1}{6 r^{2} R}\left(-4 Z^{2} X^{4}+6 Z^{[1,0,1]} X^{3}+6 X^{2} Y Z^{[0,1,1]}-2 X^{2} Z^{2} Y^{2}+6 Z X^{2} X^{[1,0,1]}-6 X Y Z Z^{[1,1,0]}\right. \\
& \left.+6 X Z^{[1,0,1]} Y^{2}-3 Y^{2} Z Z^{[0,2,0]}+6 Y^{3} Z^{[0,1,1]}-Z^{2} Y^{4}\right) \\
& Z_{R}^{[1,1,0]}=\frac{1}{6 r^{2} R^{2}}\left(6 Z^{2} X Y X^{[1,0,1]}-6 Z X Y^{2} X^{[1,1,0]}-3 Z X^{2} Y X^{[0,2,0]}+6 Z^{2} Y^{2} Y^{[1,0,1]}\right. \\
& -4 Z X^{5} Y+Z Y^{5} X+6 X^{4} Z^{[1,1,0]}-6 Y^{4} Z^{[1,1,0]}-6 Z Y Z^{[1,0,1]} X^{2}+6 Z X Z^{[0,1,1]} Y^{2} \\
& +3 Z^{2} X Y Z^{[0,2,0]}-6 Z X^{3} X^{[1,1,0]}-3 X^{3} Y^{3} Z+12 X^{3} Y X^{[1,0,1]}+12 X Y^{3} X^{[1,0,1]} \\
& -6 Z Y^{3} Z^{[1,0,1]}+6 X^{3} Y Z^{[0,2,0]}+6 X Y^{3} Z^{[0,2,0]}+6 Z X^{3} Z^{[0,1,1]} \\
& \left.-6 X^{3} Z^{3} Y+6 X^{2} Z^{2} Z^{[1,1,0]}+6 X^{2} Z^{2} Y^{[1,0,1]}-3 Z Y^{3} X^{[0,2,0]}-3 Z^{3} Y^{3} X\right) \\
& Y_{R}^{[1,0,1]}=-\frac{1}{6 r^{2} R^{2}}\left(-6 X^{4} Y^{[1,0,1]}-12 Z X Y^{2} X^{[1,1,0]}+6 Z^{2} Y^{2} Y^{[1,0,1]}-6 Y^{4} Z^{[1,1,0]}-6 Z X^{2} Y X^{[0,2,0]}\right. \\
& -6 Y^{2} Z^{[1,1,0]} X^{2}-12 Z Y Z^{[1,0,1]} X^{2}+6 Y^{2} Z^{2} Z^{[1,1,0]}-12 Y^{2} X^{2} Y^{[1,0,1]}-6 Y^{4} Y^{[1,0,1]} \\
& -6 Z Y^{3} X^{[0,2,0]}-12 Z X^{3} X^{[1,1,0]}-3 Z^{3} Y^{3} X+9 X^{3} Y^{3} Z+4 Z X^{5} Y-12 Z Y^{3} Z^{[1,0,1]} \\
& +12 Z X^{3} Z^{[0,1,1]}+3 X Y^{3} Z^{[0,2,0]}+5 Z Y^{5} X-3 Z^{2} X Y Z^{[0,2,0]}+12 Z X Z^{[0,1,1]} Y^{2}-6 Z^{2} X Y X^{[1,0,1]} \\
& \left.+6 X Y^{3} X^{[1,0,1]}+6 X^{3} Y X^{[1,0,1]}+6 X^{2} Z^{2} Y^{[1,0,1]}+3 X^{3} Y Z^{[0,2,0]}\right) \\
& X_{R}^{[1,1,0]}=\frac{1}{6 r R^{2}}\left(-Y^{5} X+3 Z^{2} Y^{3} X-6 Z Y^{2} Y^{[1,0,1]}-6 Z X^{2} Y^{[1,0,1]}-6 Z Y^{2} Z^{[1,1,0]}-2 X^{5} Y\right. \\
& +6 X^{3} X^{[1,1,0]}+3 X Y Z Z^{[0,2,0]}+3 X^{2} Y X^{[0,2,0]}+6 X Y Z X^{[1,0,1]}+6 X Y^{2} X^{[1,1,0]} \\
& \left.+3 Y^{3} X^{[0,2,0]}-3 X^{3} Y^{3}-6 Z^{2} Y Z^{[1,0,1]}+6 Z^{2} X Z^{[0,1,1]}\right), \\
& X_{R}^{[1,0,1]}=\frac{1}{6 r R}\left(-2 Z X^{4}+6 X^{2} X^{[1,0,1]}+2 Y^{2} X^{2} Z+2 X^{2} Z^{3}-6 X Y Z^{[1,1,0]}-6 Z X Z^{[1,0,1]}\right. \\
& \left.-6 Z Y Z^{[0,1,1]}+2 Y^{2} Z^{3}+Y^{4} Z-3 Z^{[0,2,0]} Y^{2}\right) \\
& X_{R}^{[0,2,0]}=-\frac{1}{3 r^{2} R}\left(-X^{4} Y^{2}-Y^{4} X^{2}-3 X Y^{2} X^{[0,2,0]}-3 X^{2} Z^{2} Y^{2}+6 Y^{2} Z X^{[1,0,1]}+6 X Y Z Z^{[1,1,0]}\right. \\
& \left.-3 Z X^{2} Z^{[0,2,0]}+6 X^{2} Y X^{[1,1,0]}-3 X^{3} X^{[0,2,0]}+6 Y^{3} X^{[1,1,0]}\right) \text {. }
\end{aligned}
$$




\section{References}

[1] D. Aouada, S. Feng, and H. Krim. Statistical analysis of the global geodesic function for 3D object classification. In proceedings of ICASSP, Honolulu, HI, 2007.

[2] M. Boutin. Numerically invariant signature curves. Int. J. Computer Vision, 40:235-248, 2000 .

[3] E. Calabi, P.J. Olver, C. Shakiban, A. Tannenbaum, and S. Haker. Differential and numerically invariant signature curves applied to object recognition. Int. J. Computer Vision, 26:107-135, 1998.

[4] É. Cartan. La méthode du repère mobile, la théorie des groupes continus, et les espaces généralisés, volume 5 of Exposés de Géométrie. Hermann, Paris, 1935.

[5] H. Derksen and G. Kemper. Computational invariant theory. Invariant Theory and Algebraic Transformation Groups, I. Springer-Verlag, Berlin, 2002. Encyclopaedia of Mathematical Sciences, 130.

[6] O. Faugeras. Cartan's moving frame method and its application to the geometry and evolution of curves in the Euclidean, affine and projective planes. Application of Invariance in Computer Vision, J.L Mundy, A. Zisserman, D. Forsyth (eds.) Springer-Verlag Lecture Notes in Computer Science, 825:11-46, 1994.

[7] M. Fels and P. J. Olver. Moving Coframes. II. Regularization and Theoretical Foundations. Acta Appl. Math., 55:127-208, 1999.

[8] S. Feng, D. Aouada, H. Krim, and I. Kogan. 3D mixed invariant and its application on object classification. In proceedings of ICASSP, Honolulu, HI, 2007.

[9] S. Feng, I. Kogan, and H. Krim. Integral invariants for 3D curves: an Inductive Construction. In proceedings of ISET/SPIE joint symposium, San Jose, CA, 2007.

[10] S. Feng, H. Krim, and I.A. Kogan. 3D Face Recognition using Euclidean Integral Invariants Signature. In proceedings of of the 14th Workshop on Signal Processing, pages 156-160, Honolulu, HI, 2007.

[11] M. L. Green. The moving frame, differential invariants and rigidity theorems for curves in homogeneous spaces. Duke Math. Journal, 45:735-779, 1978.

[12] P. A. Griffiths. On Cartan's method of Lie groups as applied to uniqueness and existence questions in differential geometry. Duke Math. Journal, 41:775-814, 1974.

[13] C. Hann and M. Hickman. Projective curvature and integral invariants. Acta applicandae mathematicae, 74:177-193, 2002.

[14] E. Hubert and I. A. Kogan. Smooth and algebraic invariants of a group action: Local and global construction. Foundation of Computational Math. J., 7:4:345-383, 2007. 
[15] E. Hubert and I.A. Kogan. Rational invariants of an algebraic group action: Construction and rewriting. Journal of Symbolic Computations, 42:203-217, 2007.

[16] D. Jensen. Higher order contact of submanifolds of homogeneous spaces, volume 610 of Lecture Notes in Mathematics. Springer-Verlag, Berlin-New York, 1977.

[17] I. A. Kogan. Two algorithms for a moving frame construction. Canad. J. Math., 55:266-291, 2003.

[18] W. Y. Lin, N. Boston, and Y. H. Hu. Summation invariant and its application to shape recognition. In Proc. of ICASSP, 2005.

[19] S. Manay, D. Cremers, B. Hong, A. Yezzi, and S. Soatto. Shape matching via integral invariants. IEEE Transactions on Pattern Analysis and Machine Intelligence, 28(10), 2006.

[20] S. Manay, A. Yezzi, B. Hong, and S. Soatto. Integral invariant signatures. In Proc. of the $E C C V, 2004$.

[21] J. L. Mundy and A. Zisserman, editors. Geometric invariance in computer vision. Artificial Intelligence. MIT Press, Cambridge, MA, 1992.

[22] J. L. Mundy, A. Zisserman, and D. Forsyth, editors. Application of invariance in computer vision. Lecture Notes in Computer Science. Springer-Verlag, 1992.

[23] P. J. Olver. Joint invariant signatures. Found. Comp. Math, 1:3-67, 2001.

[24] P.J. Olver, G. Sapiro, and A. Tannenbaum. Invariant geometric evolutions of surfaces and volumetric smoothing. SIAM J. Appl. Math., 57:176-194, 1997.

[25] J. Sato and R. Cipolla. Affine integral invariants for extracting symmetry axes. Image and Vision Computing, 15:627-635, 1997.

[26] B. Sturmfels. Algorithms in Invariant Theory. Springer-Verlag, Vienna, 1993.

[27] G. Taubin and D. Cooper. Object recognition based on moment (or algebraic) invariants. Geometric Invariance in Computer Vision, J.L. Mundy and A. Zisserman (eds), MIT Press, pages 375-397, 1992.

[28] L. Van Gool, T. Moons, E. Pauwels, and A. Oosterlinck. Semi-differential invariants. Geometric Invariance in Computer Vision, J.L. Mundy and A. Zisserman (eds), MIT Press, pages 157-192, 1992.

[29] I. Weiss. Geometric invariants and object recognition. Acta applicandae mathematicae, 10:207-231, 1993.

[30] D. Xu and H. Li. 3-d affine moment invariants generated by geometric primitives. In proceedings of $I C P R$, pages $544-547,2006$.

[31] Princeton Shape Benchmark database: http://shape.cs.princeton.edu/benchmark/, 2005. 